\title{
Bitopertin, a selective oral GLYT1 inhibitor, improves anemia in a mouse model of $\boldsymbol{\beta}$-thalassemia
}

\author{
Alessandro Matte, ${ }^{1}$ Enrica Federti, ${ }^{1}$ Michael Winter, ${ }^{2}$ Annette Koerner, ${ }^{2}$ Anja Harmeier, ${ }^{2}$ \\ Norman Mazer, ${ }^{2}$ Tomas Tomka, ${ }^{2}$ Maria Luisa Di Paolo, ${ }^{3}$ Luigia De Falco, ${ }^{4}$ Immacolata Andolfo, ${ }^{4}$ \\ Elisabetta Beneduce, ${ }^{1}$ Achille Iolascon, ${ }^{4}$ Alejandra Macias-Garcia, ${ }^{5}$ Jane-Jane Chen, ${ }^{5}$ Anne Janin, ${ }^{6,7,8}$ \\ Christhophe Lebouef, ${ }^{6,7,8}$ Franco Turrini, ${ }^{9}$ Carlo Brugnara, ${ }^{10}$ and Lucia De Franceschi ${ }^{1}$ \\ 'Department of Medicine, University of Verona and Azienda Ospedaliera Universitaria Verona, Policlinico GB Rossi, Verona, \\ Italy. ${ }^{2}$ Pharmaceutical Research and Early Development, Roche Innovation Center Basel, F. Hoffmann-La Roche Ltd, \\ Basel, Switzerland. ${ }^{3}$ Department of Molecular Medicine, University of Padova, Padova, Italy. ${ }^{4}$ Department of Molecular \\ Medicine and Medical Biotechnology, University Federico II and CEINGE, Naples, Italy. ${ }^{5}$ Institute for Medical Engineering \\ and Science, Massachusetts Institute of Technology, Cambridge, Massachusetts, USA. 6 INSERM, U1165, Paris, France. \\ ${ }^{7}$ Université Paris 7 - Denis Diderot, Paris, France. ${ }^{8}$ AP-HP, Hôpital Saint-Louis, Paris, France. ${ }^{9}$ Department of Oncology, \\ University of Torino, Torino, Italy. ${ }^{10}$ Department of Laboratory Medicine, Boston Children's Hospital, Harvard Medical \\ School, Boston, Massachusetts, USA.
}

Anemia of $\beta$-thalassemia is caused by ineffective erythropoiesis and reduced red cell survival. Several lines of evidence indicate that iron/heme restriction is a potential therapeutic strategy for the disease. Glycine is a key initial substrate for heme and globin synthesis. We provide evidence that bitopertin, a glycine transport inhibitor administered orally, improves anemia, reduces hemolysis, diminishes ineffective erythropoiesis, and increases red cell survival in a mouse model of $\boldsymbol{\beta}$-thalassemia ( $\boldsymbol{H b}^{\text {th } 3 /+}$ mice). Bitopertin ameliorates erythroid oxidant damage, as indicated by a reduction in membrane-associated free $\alpha$-globin chain aggregates, in reactive oxygen species cellular content, in membrane-bound hemichromes, and in heme-regulated inhibitor activation and elF2 $\alpha$ phosphorylation. The improvement of $\beta$-thalassemic ineffective erythropoiesis is associated with diminished mTOR activation and Rab5, Lamp1, and p62 accumulation, indicating an improved autophagy. Bitopertin also upregulates liver hepcidin and diminishes liver iron overload. The hematologic improvements achieved by bitopertin are blunted by the concomitant administration of the iron chelator deferiprone, suggesting that an excessive restriction of iron availability might negate the beneficial effects of bitopertin. These data provide important and clinically relevant insights into glycine restriction and reduced heme synthesis strategies for the treatment of $\beta$-thalassemia.

Authorship note: EF and MW

Conflict of interest: CB discloses a 2013-2018 consulting agreement with Hoffman-La Roche. LDF discloses research funding by Hoffman-La Roche for the period 2015-2018. $\mathrm{MW}, \mathrm{AK}, \mathrm{AH}$, and NM are employees of Hoffman-La Roche. TT was an employee of Hoffman-La Roche until October 31, 2018.

Copyright: (c) 2019, American Society for Clinical Investigation.

Submitted: May 13, 2019

Accepted: October 2, 2019

Published: November 14, 2019.

Reference information: JCI Insight. 2019;4(22):e130111.

https://doi.org/10.1172/jci.

insight.130111

\section{Introduction}

Ineffective erythropoiesis is a distinguishing feature of $\beta$-thalassemias ( $\beta$-thals), common inherited red cell disorders due to the absence of, or the reduction in, synthesis of $\beta$-globin chains (1-6). In $\beta$-thal, the excess of unpaired $\alpha$-globin chains and the decoupling between heme and $\beta$-globin chain synthesis with relative free heme excess results in enhanced production of reactive oxygen species (ROS) with severe cellular oxidative damage, leading to a block in cell maturation at the basophilic erythroblast stage, increased cell apoptosis, and ineffective erythropoiesis. ROS continue to inflict damage on the maturing reticulocytes and erythrocytes, causing hemolysis and a dramatically shortened red cell survival in the peripheral circulation $(5,7)$.

Several mechanisms play a key role in modulating ROS-related cytotoxic effects during erythroid maturation in $\beta$-thal (8-11). The heme-regulated eIF2 $\alpha$ kinase (HRI) is highly sensitive to intracellular erythroid heme concentrations and is a key heme-sensing regulator of globin chain production. HRI can also be activated by denatured proteins, such as the $\alpha$-globin aggregates seen in $\beta$-thal, and is necessary for the cytoplasmic unfolded protein response (9). HRI also regulates responses to increased ROS production. Absence of HRI worsens anemia in a $\beta$-thal mouse model (8-11). Peroxiredoxin-2 (Prx2) binds heme 
with high affinity and reduces its pro-oxidant effect during erythropoiesis (12-15). Both HRI and Prx2 are dynamically modulated in $\beta$-thal erythroblasts to favor erythroid survival and maturation $(10,11,13)$.

Previous studies have shown that modulation of heme homeostasis might affect erythropoiesis (16) and beneficially affect $\beta$-thal erythroid maturation (17). In erythroblasts, glycine uptake via the glycine carrier system, GlyT1, is a key determinant of the rate of heme biosynthesis and bioavailability $(18,19)$. Indeed, mice genetically lacking GlyT1 display a hypochromic microcytic anemia associated with a $25 \%$ reduced mean corpuscular hemoglobin $(\mathrm{MCH})$ and mean corpuscular volume (MCV), supporting the importance of GlyT1 in providing glycine for heme biosynthesis during erythroid maturation (16).

Bitopertin is an oral selective inhibitor of GlyT1 that was originally developed to treat negative symptoms of patients with schizophrenia (20-23). Although efficacy of bitopertin in schizophrenia could not be proved in several randomized, double-blind, placebo-controlled, phase III trials, a dose-dependent microcytic hypochromic erythropoiesis with reduction in hemoglobin $(\mathrm{Hb})$ values was consistently observed in bitopertin-treated subjects. This indicated the ability of bitopertin to inhibit GlyT1-mediated glycine transport during normal erythropoiesis, generating a "heme-restricted" environment (20, 21). Heme-sensing HRI has been shown to be responsible for microcytic hypochromic anemia of iron deficiency (10).

Here, we sought to determine the role of "heme restriction" on $\beta$-thal ineffective erythropoiesis. Our goal was to examine whether bitopertin could influence ineffective erythropoiesis and improve red cell survival and anemia in an established mouse model for $\beta$-thal. We report that treatment of $\beta$-thal mice with oral bitopertin reduces ineffective erythropoiesis and ameliorates multiple markers of oxidant damage, resulting in improved red cell survival and reduction of anemia and hemolysis. Coadministration of an oral iron chelator reduces the beneficial effect of bitopertin in $\beta$-thalassemic mice.

\section{Results}

Bitopertin improves anemia of $\beta$-thalassemic mice. In $\beta$-thalassemic $\left(\mathrm{B} 6 ; 129 \mathrm{P} 2-\mathrm{Hbb}-\mathrm{b} 1^{\mathrm{tm} 1 \mathrm{Unc}} \mathrm{Hbb}-\mathrm{b} 2^{\mathrm{tm} 1 \mathrm{Unc} / \mathrm{J}}\right)$ heterozygous; $H b b^{t h 3 /+}$ ) mice, oral bitopertin (at the dosage of $30 \mathrm{mg} / \mathrm{kg} / \mathrm{d}$, given once daily via gavage) improved red cell morphology compared with vehicle-treated animals (Figure 1A). This was associated with increased $\mathrm{Hb}$ at 2 weeks of treatment, then with reaching a plateau, which was maintained until the end of the bitopertin treatment period at day 28 (Figure 1B). A significant increase in $\mathrm{Hb}$ was also observed in $\mathrm{Hbb}^{\text {th } 3 /+}$ mice treated with bitopertin at lower dosages of either 3 or $10 \mathrm{mg} / \mathrm{kg} / \mathrm{d}$ (Supplemental Figure 1A; supplemental material available online with this article; https://doi.org/10.1172/jci.insight.130111DS1). In Hbb ${ }^{\text {th } 3 /+}$ mice treated with $30 \mathrm{mg} / \mathrm{kg} / \mathrm{d}$ bitopertin, the increased $\mathrm{Hb}$ values were associated with an increase in $\mathrm{RBC}$ counts (Supplemental Figure 1B) and a slight but significant decrease in MCV (Supplemental Figure 1B) with a slightly increased $\mathrm{MCH}$ (Figure 1B, days 7 and 14 only). Significant decreases in absolute reticulocyte counts and in circulating erythroblasts were also found in $\mathrm{Hbb}^{\text {th3/+}}$ mice treated with bitopertin at dosages of either 30 or $10 \mathrm{mg} / \mathrm{kg} / \mathrm{d}$ for 28 days (Figure 1B and Supplemental Figure 1C). No major change in these parameters was observed in $\mathrm{Hbb}^{\text {th } 3 /+}$ mice treated with a lower bitopertin dose of $3 \mathrm{mg} / \mathrm{kg} / \mathrm{d}$.

The amelioration of anemia and red cell indices was associated with a significant reduction in $\alpha$-globin membrane precipitates and an increase in the fraction of soluble $\mathrm{Hb}$ in red cells from bitopertin-treated $\mathrm{Hb}^{t h 3 /+}$ mice compared with vehicle-treated animals (Figure 1, C and D). This was associated with a significant decrease in plasma total bilirubin and lactate dehydrogenase, known markers of hemolysis (Figure 1E). In agreement with these results, we found a marked reduction in plasma erythropoietin (EPO) in bitopertin-treated $\mathrm{Hbb}^{\text {th3/+}}$ mice compared with vehicle-treated animals (Supplemental Figure 1D).

Taken together, these results indicate that bitopertin ameliorates anemia of $\mathrm{Hbb}^{\text {th } 3 /+}$ mice. WT mice showed hematologic changes similar to those previously reported in healthy rats, with the appearance of mild hypochromic microcytic erythrocytes in the absence of anemia (20).

Bitopertin reduces $\beta$-thalassemic ineffective erythropoiesis. In $H b b^{\text {th } 3 /+}$ mice, bitopertin $(30 \mathrm{mg} / \mathrm{kg} / \mathrm{d})$ significantly decreased extramedullary erythropoiesis as supported by (a) the reduction in the spleen/body weight ratio, (b) the decrease of iron accumulation in the spleen assessed with Pearl's staining, and (c) the reduction in spleen erythropoietic activity and in bone marrow total erythroblasts (Figure 2, A-C, and Supplemental Figure 2, A and B). This was associated with improvement in morphology of $\beta$-thal erythroblasts, as supported by the presence of more regular nuclear shape, increased nuclear condensation, reduction in the number of binucleated cells, and improved enucleation as observed in some orthochromatic erythroblasts (Figure 2D).

Because $\beta$-thal is characterized by ineffective erythropoiesis with blockage in erythroid maturation associated with expansion of more immature erythroblasts, we evaluated the ratio between orthochromatic 
A Baseline
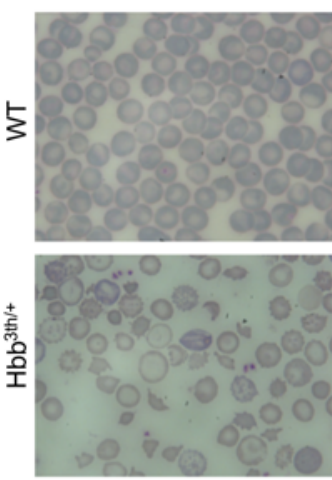

Bitopertin 14d
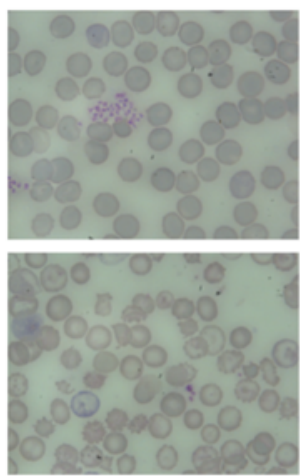

Bitopertin 28d
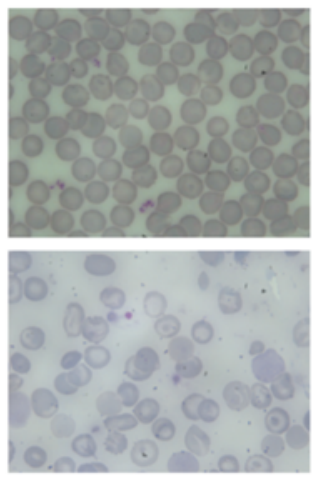

C

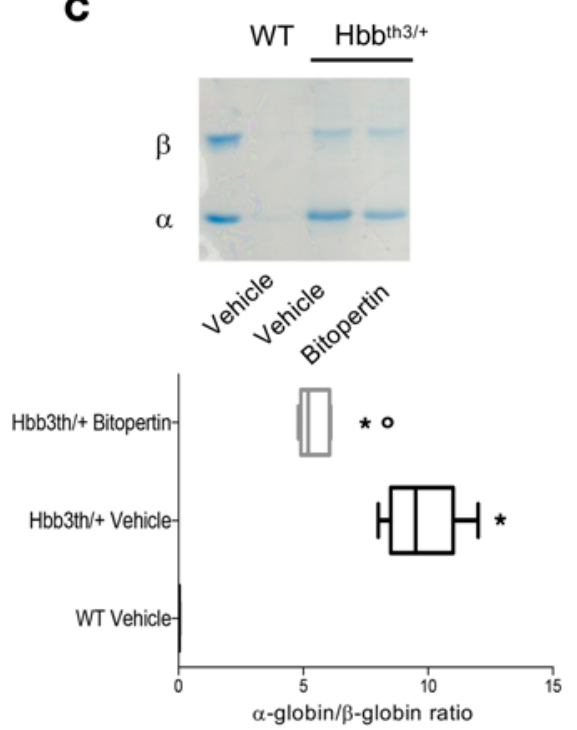

$(\mathrm{OD})$

\section{B}

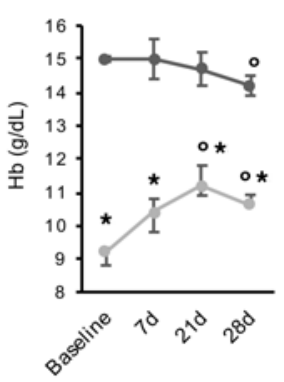

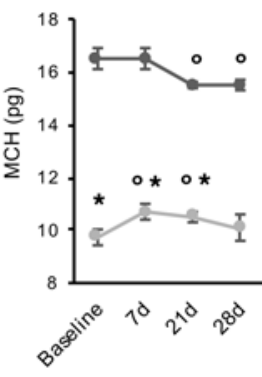

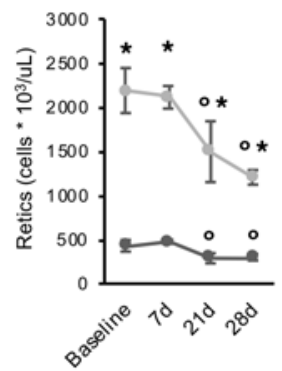

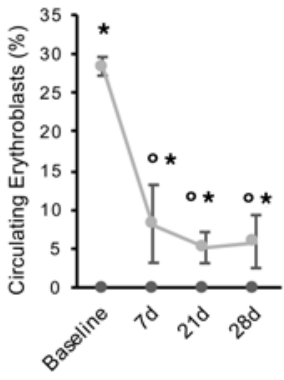
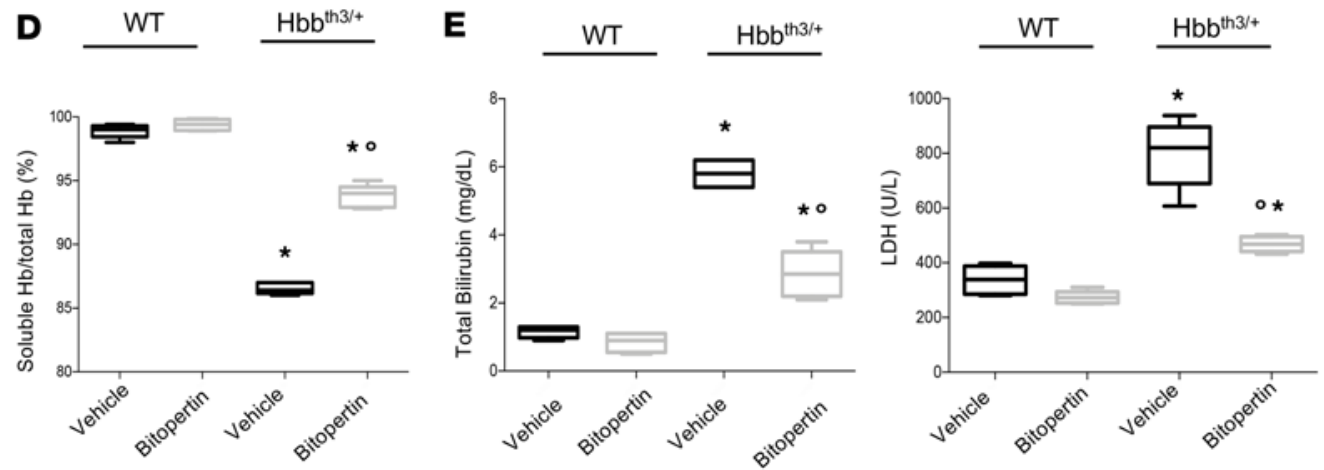

Figure 1. Bitopertin treatment improves chronic hemolytic anemia of a mouse model for $\beta$-thalassemia. Data from WT and $\mathrm{Hb} b^{3 t h /+}$ mice treated with either vehicle or bitopertin $30 \mathrm{mg} / \mathrm{kg} / \mathrm{d}$ are presented. (A) Normal erythrocyte blood smear morphology in bitopertin-treated WT mice. Hbb ${ }^{\text {th }} / \mathrm{t}$ showed typical hypochromic red cells, marked polychromasia, and various forms of fragmented erythrocytes (poikilocytes) associated with stressed erythropoiesis and hemolysis. Significant improvement of poikilocytosis after 2 and 4 weeks of treatment with bitopertin. May-Grünwald-Giemsa-stained smears were imaged under oil at original magnification $\times 100$ using a Panfluor objective with 1.30 numeric aperture on a Nikon Eclipse DS-5M camera and processed with Digital Slide (DS-L1) Nikon. One representative image from the other 6 with similar results is presented. (B) Hemoglobin (Hb), mean corpuscular Hb $(\mathrm{MCH})$, reticulocyte count, and circulating erythroblasts in WT $(n=6)$ and $\mathrm{Hbb}^{3 \mathrm{th} /+}$ mice $(n=6)$ mice under either vehicle or bitopertin treatment. Data are presented as mean $\pm \mathrm{SD}$; ${ }^{*} P<0.05$ compared with WT mice; ${ }^{\circ} P<0.05$ compared with baseline values; 2 -way ANOVA with Tukey's test for multiple comparisons. (C) Upper: Triton acid urea gel electrophoresis of red cell membrane from WT and $\mathrm{Hbb}^{\text {th } 3 /+}$ mice under either vehicle or bitopertin treatment (28 days). Lower: Quantification of gel bands (OD) expressed as $\alpha$-globin to $\beta$-globin ratio to Hb. Data are shown as median and minimum/maximum ( $n=6$ ); ${ }^{*} P<0.02$ compared with WT animals; ${ }^{\circ} P<0.05$ compared with vehicle-treated $H b b^{\text {th } 3 /+}$ mice; 2-way ANOVA with Holm-Šídák test for multiple comparisons. (D) Measurement of total and soluble $\mathrm{Hb}$ by Drabkin's method in hemolysates from $\mathrm{Hbb}^{\text {th } 3 /+}$ mice under either vehicle or bitopertin treatment (30 $\mathrm{mg} / \mathrm{kg} / \mathrm{d}, 28$ days). Data are shown as mean $\pm \mathrm{SD}(n=6) ;{ }^{*} P<0.05$ compared with vehicle-treated $H b b^{\text {th } 3 /+}$ mice. ${ }^{\circ} P<0.05$ compared with vehicle-treated group. (E) Plasma total bilirubin and lactate dehydrogenase in WT $(n=4)$ and $H b b^{3 t h /+}$ mice $(n=4)$ mice under either vehicle or bitopertin treatment (30 $\mathrm{mg} / \mathrm{kg} / \mathrm{d}, 28$ days). Data are presented as median and minimum/maximum; ${ }^{*} P<0.05$ compared with WT mice; ${ }^{\circ} P<0.05$ compared with vehicle-treated group; Mann-Whitney $U$ test with multiple-comparisons corrections. 
A
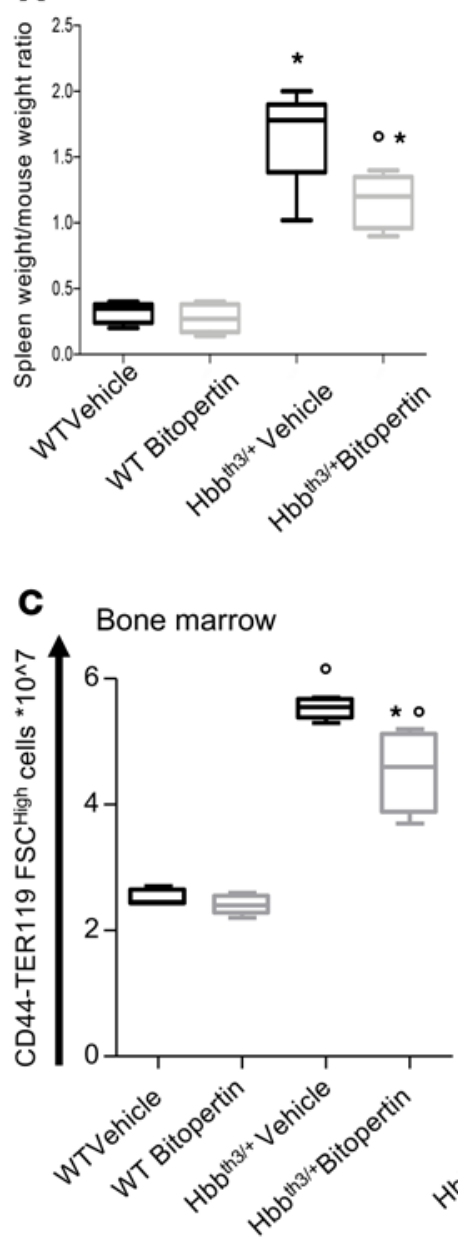

B
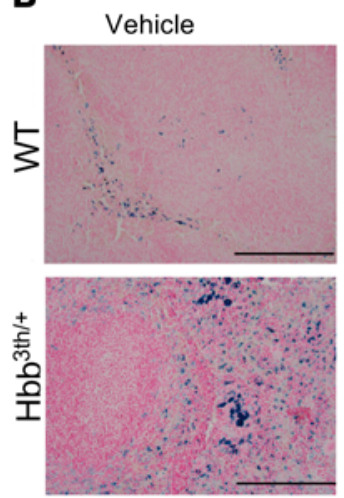

Spleen
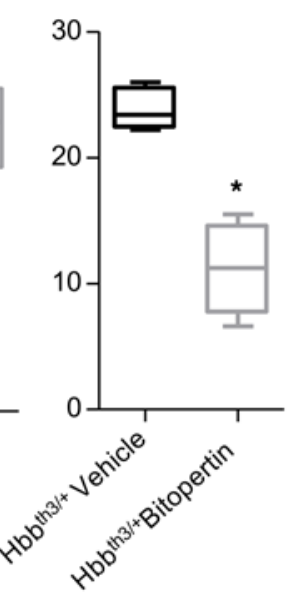

E Spleen
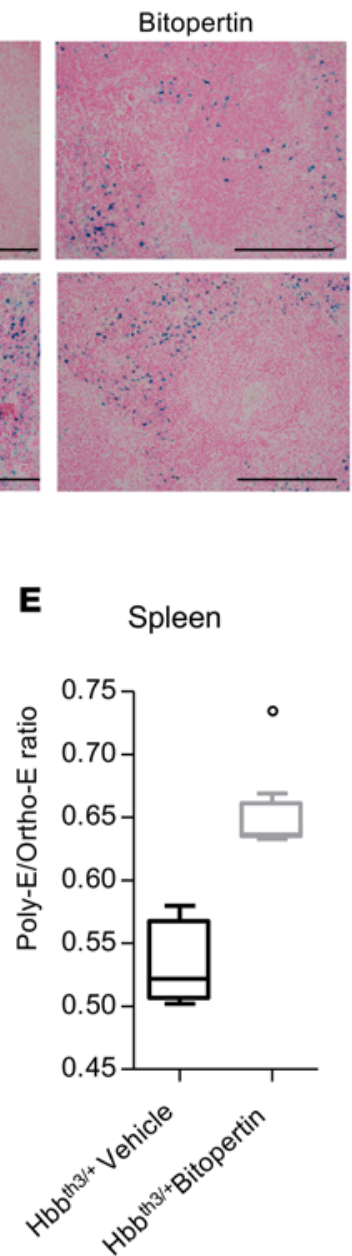

D $\mathrm{Hbb}^{3 \mathrm{~h} / \mathrm{P}}$ Poly-EB $\quad \mathrm{Hbb}^{3 \mathrm{~h} /+}$ Ortho-EB
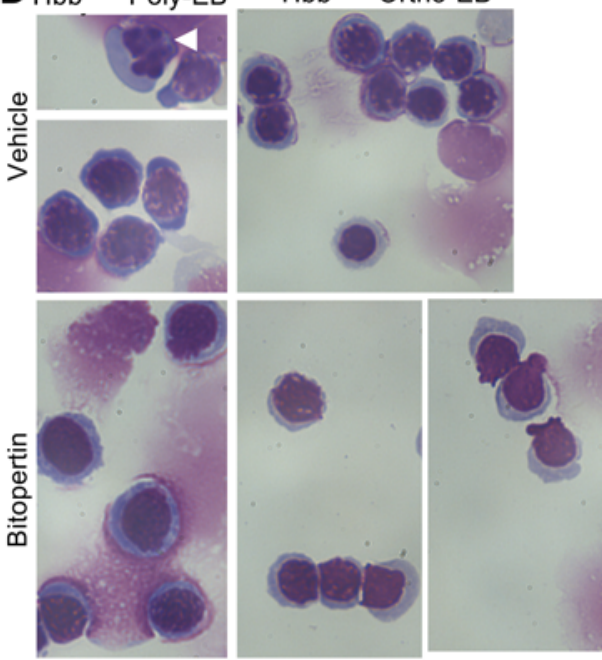

$\square$ Abnormal nuclear shaped E. Binuclear E.

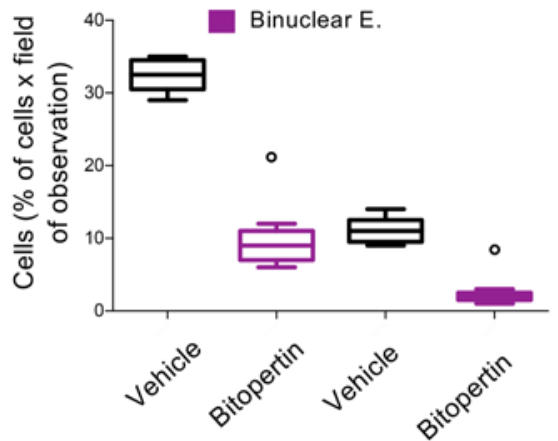

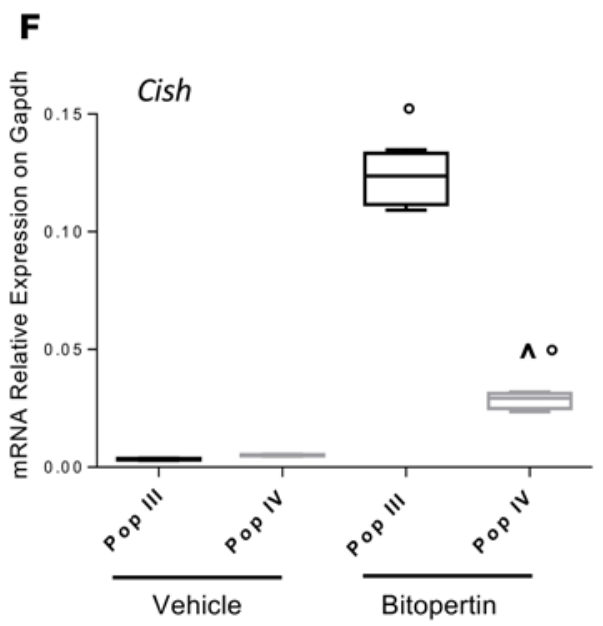
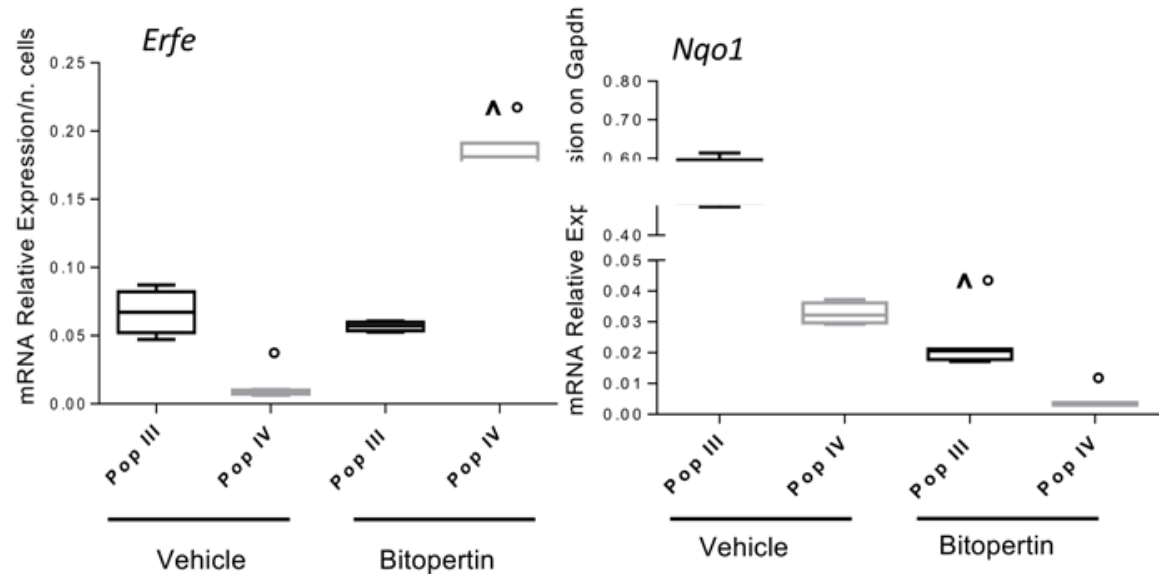
Figure 2. Bitopertin reduces $\beta$-thalassemic ineffective erythropoiesis. (A) Spleen weight/mouse weight ratio in WT and $H b b^{\text {th } 3 /+}$ mice with and without bitopertin treatment $\left(30 \mathrm{mg} / \mathrm{kg} / \mathrm{d}, 28\right.$ days). Data are presented as median and minimum/maximum $(n=6) ;{ }^{*} P<0.05$ compared with WT mice; ${ }^{\circ} P<0.05$ compared with vehicle-treated group; Mann-Whitney $U$ test with multiple-comparisons corrections. (B) Iron staining (Pearl's Prussian blue) in spleens from WT and $\mathrm{Hbb}^{\text {th } 3 /+}$ mice with and without bitopertin treatment (30 mg/kg/d, 28 days). One representative image from the other 6 with similar results is presented. Original magnification, $\times 200$. See Supplemental Figure 2A for quantitative results of iron staining on Pearl's Prussian blue. (C) Flow cytometric analysis combining CD44-Ter119 and cell size marker strategy (CD44+Ter119+Fschi) on bone marrow and spleens from WT and $H b b^{\text {th } 3 /+}$ mice with and without bitopertin treatment (28 days). Data are presented as median and minimum/maximum $(n=3) ;{ }^{*} P<0.05$ compared with WT mice; ${ }^{\circ} P<0.05$ compared with vehicle-treated mice; 2-way ANOVA with Holm-Šídák test for multiple comparisons. (D) Upper: Morphology of sorted erythroid cells from $\mathrm{Hbb}^{\text {th } 3 /+}$ mice with and without bitopertin treatment ( $30 \mathrm{mg} / \mathrm{kg} / \mathrm{d}, 28$ days). Cytospins were stained with May-Grünwald-Giemsa. Cells were imaged under oil at original magnification $\times 100$ using a Panfluor objective with 1.30 numeric aperture on a Nikon Eclipse DS-5M camera and processed with Digital Slide (DSL1) Nikon. We show 1 representative image from a total of 6 . Erythroblasts with abnormal nuclear shape and binuclear erythroblasts (white arrowhead) from $\mathrm{Hbb}^{\text {th }} \mathrm{h/+}$ mice evaluated on cytospin stained with May-Grünwald-Giemsa. Lower: Data are presented as median and minimum $/ \mathrm{maximum}(n=6) ;{ }^{\circ} P$ $<0.05$ compared with vehicle-treated group; 2-way ANOVA with Holm-Šídák test for multiple comparisons. (E) Box plot showing the ratio between polychromatic/orthochromatic erythroblasts used as a maturation index in spleens analyzed by flow cytometry from $\mathrm{Hb}^{b^{\text {th }} /++}$ mice with and without bitopertin treatment ( $30 \mathrm{mg} / \mathrm{kg} / \mathrm{d}, 28$ days). Data are presented as median and minimum/maximum $(n=6) ;{ }^{\circ} P<0.05$ compared with standard diet; 2 -way ANOVA with Holm-Šídák test for multiple comparisons. (F) mRNA expression by real-time reverse transcription PCR (qRT-PCR) of Cish, NAD(P)H quinone-1 (Nqo1), and erythroferrone (Erfe; Fam132b normalized on number of sorted cells in each subpopulation) genes on sorted polychromatic erythroblasts (Pop III) and orthochromatic erythroblasts (Pop IV) from bone marrow of WT and $\mathrm{Hbb}^{\text {th } 3 /+}$ mice with and without bitopertin treatment (30 mg/kg/d, 28 days). Experiments were performed in triplicate. Data are presented as median and minimum/maximum; ${ }^{\circ} P<0.05$ compared with vehicle; ${ }^{\wedge} P<0.05$ Pop IV vs. Pop III; Mann-Whitney $U$ test with multiple-comparison corrections.

(more mature) and polychromatic (more immature) erythroblasts, expressed as erythroid maturation index $(13,24)$. In $\mathrm{Hbb}^{\mathrm{th} /+}$ mice, bitopertin treatment $(30 \mathrm{mg} / \mathrm{kg} / \mathrm{d})$ induced a significant increase in maturing erythroblasts in the spleen (Figure 2E) but not in bone marrow (data not shown). To further analyze the impact of bitopertin on $\beta$-thal erythropoiesis, we used the CD71-TER119 gating strategy (Supplemental Figure 2C). This allowed us to observe a significant reduction in bone marrow basophilic (II) and polychromatic (III) erythroblasts and a marked increase in orthochromatic (IV) erythroblasts in $\mathrm{Hb}^{\text {th } 3 /+}$ mice treated with $30 \mathrm{mg} / \mathrm{kg} / \mathrm{d}$ bitopertin (Supplemental Figure 2C). A reduction of all maturating populations was observed in the spleen, reflecting an overall decrease in extramedullary erythropoiesis in $\mathrm{Hbb}^{t h 3 /+}$ mice treated with $30 \mathrm{mg} / \mathrm{kg} / \mathrm{d}$ bitopertin (Supplemental Figure 2C).

In the bone marrow from bitopertin-treated $\mathrm{Hbb}^{t h 3 /+}$ mice, a significant decrease in the amount of annexin $\mathrm{V}^{+}$cells present in the $\mathrm{CD} 44^{10} \mathrm{Ter} 119^{+} \mathrm{Fsc}{ }^{\mathrm{lo}}$ and $\mathrm{CD} 44-\mathrm{Ter} 119^{+} \mathrm{Fsc}^{\mathrm{lo}}$ populations was also observed (Supplemental Figure 3A).

The bitopertin-induced improvement of ineffective erythropoiesis and of its EPO responsiveness was supported by upregulation of cytokine-inducible SH2-containing protein (Cish), a gene strictly dependent on STAT5 function, and erythroferrone (Erfe), which is also linked to iron homeostasis $(12,25-27)$ (Figure $2 \mathrm{~F}$ ), and by reduction in plasma EPO levels (Supplemental Figure 1D). This was associated with downregulation of Nqo1, an antioxidant system (Figure 2F) (12, 25-27). Collectively, our data suggest that heme restriction induced by bitopertin ameliorates $\beta$-thalassemic ineffective erythropoiesis, improving EPO responsiveness and reducing oxidative stress.

Bitopertin reduces liver iron overload and oxidative stress and modulates liver hepcidin expression in a dose-dependent manner. Liver iron overload is a distinguishing feature of $\beta$-thal, a consequence of ineffective erythropoiesis and inappropriate hepcidin (Hamp) downregulation (28-30). In $H b b^{t h 3 /+}$ mice, bitopertin treatment $(30 \mathrm{mg} / \mathrm{kg} / \mathrm{d})$ significantly reduced liver iron overload in both hepatocytes and Kupffer cells compared with vehicle-treated animals (Figure 3A and Supplemental Figure 3B). A significant reduction in liver protein oxidation as assessed by OxyBlot was observed (Figure 3B), in conjunction with reduced activation of cytoprotective systems, such as heme oxygenase 1, or Nqo1 and Prx2, 2 potent antioxidant systems (Figure 3C). Bitopertin treatment resulted in upregulation of liver Hamp expression in $H b b^{t h 3 /+}$ mice (Figure 3D). This was associated with upregulation of inhibitor of DNA binding 1 (IdI), which is linked to the activation of the SMAD system (Figure 3D). No major change in either STAT3 or ERK1/2 activation was observed in bitopertin-treated $\mathrm{Hbb}^{t h 3 /+}$ mice compared with vehicle-treated animals (Supplemental Figure 3C).

To better understand the impact of bitopertin on Hamp, we administered bitopertin at the lower dose of $10 \mathrm{mg} / \mathrm{kg} / \mathrm{d}$. In $\mathrm{Hbb}^{\mathrm{th} /+}$ mice, bitopertin treatment $(10 \mathrm{mg} / \mathrm{kg} / \mathrm{d})$ was associated with amelioration of murine $\beta$-thal anemia, a reduction in splenic and bone marrow erythropoietic activity (Supplemental Figure 4, A and B). This was again associated with upregulation of Erfe in sorted erythroblasts (Supplemental Figure 5A). Bitopertin-treated $H b b^{t h 3 /+}$ mice at a dose of $10 \mathrm{mg} / \mathrm{kg} / \mathrm{d}$ exhibited downregulation of Hamp (Supplemental Figure 5B), which was associated with upregulation of Id1 (Supplemental Figure 5C) and 
a
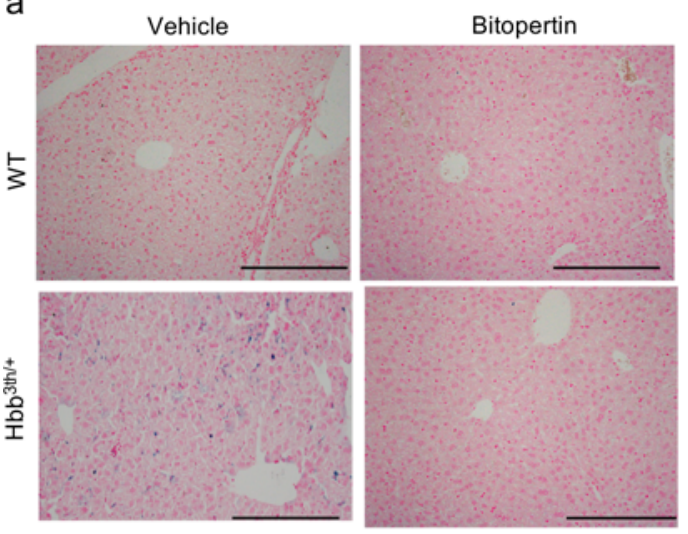

GAPDH

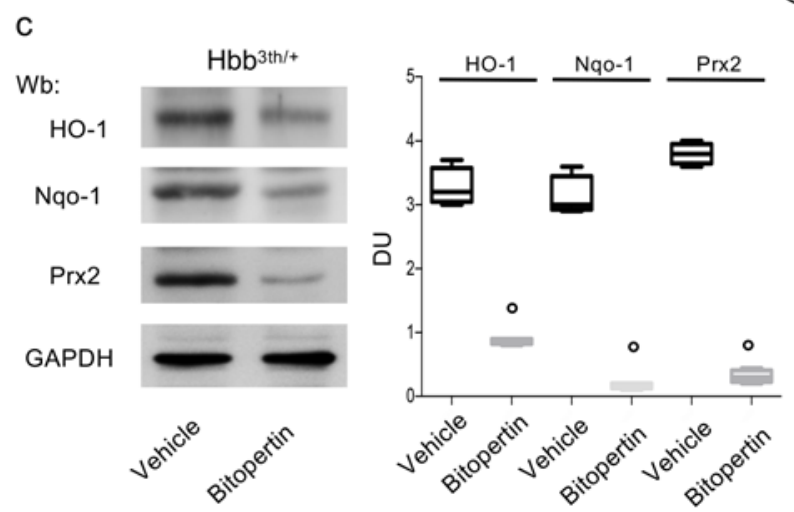

d
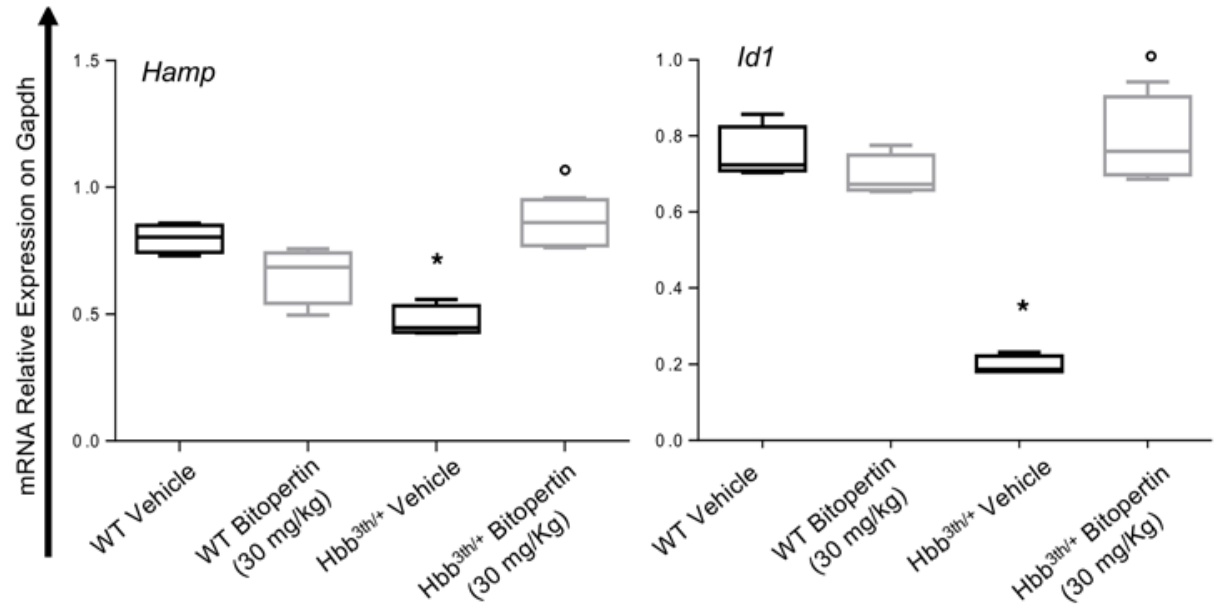
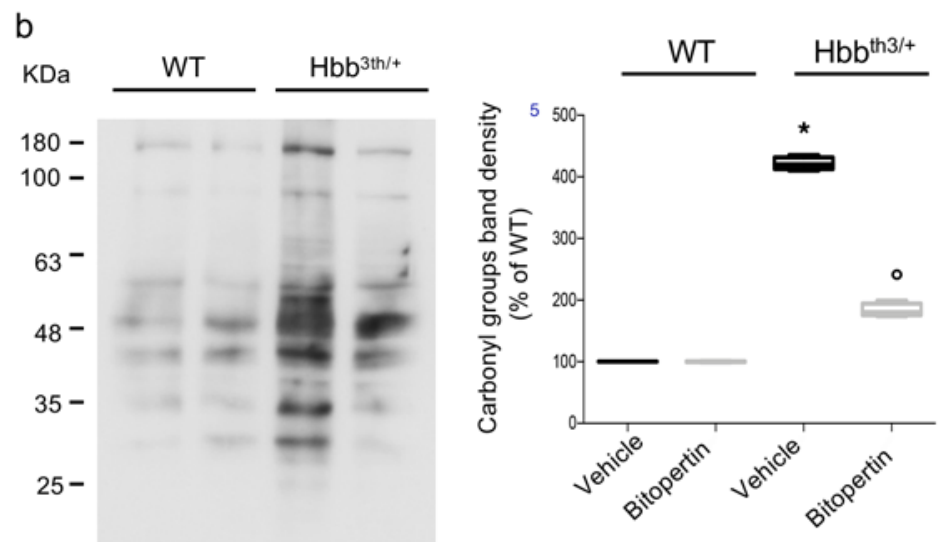
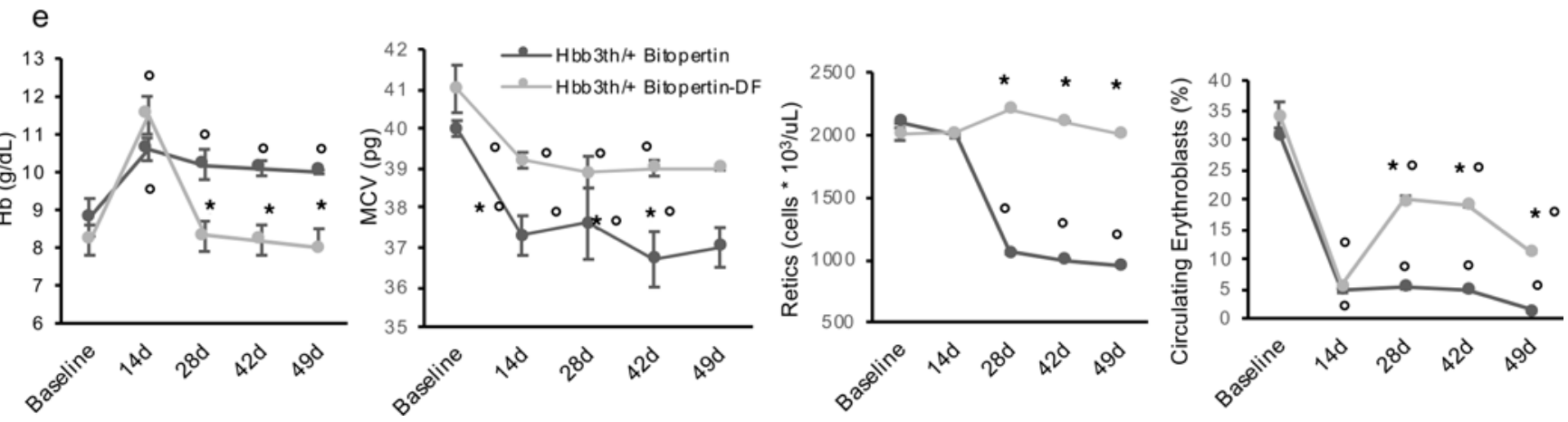
Figure 3. In $\beta$-thalassemic mice, bitopertin reduces liver iron overload and oxidative stress with associated upregulation of hepcidin. (A) Iron staining (Pearl's Prussian blue) in liver from WT and $\mathrm{Hb}^{\text {th }} 3 / \mathrm{t}$ mice with and without bitopertin treatment (28 days). One representative image from the other 3 with similar results is presented (quantification of iron staining is shown in Supplemental Figure 2E). Original magnification, $\times 200$. (B) Soluble fractions of liver from WT and $\mathrm{Hb}^{\text {th } 3 /+}$ mice with and without bitopertin treatment ( $30 \mathrm{mg} / \mathrm{kg} / \mathrm{d}, 28$ days). The samples were analyzed on 12\% SDS-PAGE and subjected to OxyBlot. The carbonylated proteins (1 mg) were detected by treating with 2,4-dinitrophenylhydrazine and blotted with anti-DNP antibody. Quantification of band area was performed by densitometry and expressed as percentage of WT (right). The data are presented as median and minimum/maximum of at least 5 experiments; ${ }^{*} P<0.05$ compared with WT; ${ }^{\circ} P<0.05$ compared with vehicle; 2 -way ANOVA with Holm-Šídák test for multiple comparisons. (C) Left: Western blot ( $\mathrm{Wb}$ ) analysis with specific antibodies against heme oxygenase (HO-1), NAD(P)H quinone-1 (Nq01), and peroxiredoxin-2 (Prx2), of liver from $\mathrm{Hbb}^{\text {th } 3 /+}$ mice with and without bitopertin treatment $(30 \mathrm{mg} / \mathrm{kg} / \mathrm{d}, 28$ days). One representative gel from 6 with similar results is presented. Right: Densitometric analysis of immunoblots is shown in the box plot. Data are presented as median and minimum/maximum; ${ }^{\circ} P<0.05$ compared with vehicle; 2-way ANOVA with Holm-Šídák test for multiple comparisons. DU, densitometric units. (D) mRNA expression by qRT-PCR of hepcidin (Hamp) and Id1 genes on liver from WT and $\mathrm{Hbb}^{\text {th } 3 /+}$ mice with and without bitopertin treatment ( $30 \mathrm{mg} / \mathrm{kg} / \mathrm{d}, 28$ days). Experiments were performed in triplicate. Error bars represent the SDs, median, and minimum/maximum; ${ }^{*} P<0.05$ compared with WT; ${ }^{\circ} P<0.05$ compared with vehicle; 2 -way ANOVA with Holm-Šídák test for multiple comparisons. (E) Hb, mean cell volume (MCV), reticulocyte count, and circulating erythroblasts in WT Hbb $b^{3 t h /+}$ mice under either bitopertin or bitopertin plus deferiprone (DFP) treatment. Data are presented as mean $\pm \mathrm{SD}$; ${ }^{*} P<0.05$ compared with bitopertin-treated mice; ${ }^{\circ} P<0.05$ compared with baseline values; Wilcoxon's signed-rank test for multiple comparisons.

reduction of activation of STAT3 and ERK1 compared with vehicle-treated animals (Supplemental Figure $5, \mathrm{D}$ and $\mathrm{E})$. A significant reduction in liver protein oxidation as assessed by OxyBlot was also observed in Bitopertin-treated $\mathrm{Hbb}^{\mathrm{th} / \mathrm{+}}$ mice at a dose of $10 \mathrm{mg} / \mathrm{kg} / \mathrm{d}$ (Supplemental Figure $5 \mathrm{~F}$ ).

Taken together, these data indicate a dose-dependent effect of bitopertin on Hamp expression, with lower doses reducing the activation of STAT3 and ERK1/2, while higher doses of bitopertin $(30 \mathrm{mg} / \mathrm{kg} / \mathrm{d})$ most likely affect the intracellular iron-driven regulation of hepcidin through the activation of the SMAD pathway, independent of Erfe.

$\beta$-Thalassemic mice treated with bitopertin and the iron chelator deferiprone show increased extramedullary erythropoiesis and worsening of anemia. In $\beta$-thal, iron chelation is part of the goal of standard treatment to prevent organ damage induced by iron overload related to both chronic transfusion regimen and chronic hemolysis. However, the presence of an iron chelator in conjunction with bitopertin treatment might worsen $\beta$-thalassemic erythropoiesis because of an additional reduction in iron availability below the minimal value required to ensure erythroid maturation.

$\beta$-Thalassemic mice were treated with bitopertin at the dosage of $30 \mathrm{mg} / \mathrm{kg} / \mathrm{d}$ in combination with deferiprone (DFP, $1.25 \mathrm{mg} / \mathrm{mL}$ ) (28). Casu et al. have previously shown in the same mouse model for $\beta$-thal that DFP did not affect erythropoiesis (28). As shown in Figure 3E, $\beta$-thalassemic mice treated with both bitopertin and DFP showed a worsening of the anemia after an early improvement at 14 days of therapy, with a higher MCV than the bitopertin control. The reduction in the elevated reticulocyte count of $\beta$-thalassemic mice treated with bitopertin was abolished in animals treated with both bitopertin and DFP (Figure 3E). In addition, we found that the stable and significant reduction in circulating erythroblasts induced by bitopertin alone could not be maintained after the first week of treatment when $\beta$-thalassemic mice were treated with bitopertin with DFP (Figure 3E). These findings suggest a worsening of ineffective erythropoiesis in bitopertin with DFP-treated $\beta$-thalassemic mice compared with $\beta$-thalassemic animals treated with bitopertin alone. Indeed, we found no major change in erythropoietic activity in $\beta$-thalassemic mice under bitopertin with DFP treatment compared with vehicle-treated animals (Supplemental Figure 6, A and B).

In circulating red cells, we confirmed the reduction of ROS in bitopertin-treated $\beta$-thalassemic mice, whereas in bitopertin with DFP-treated $\beta$-thalassemic animals, the generation of ROS was similar to that observed in vehicle-treated animals (Supplemental Figure 6C). In agreement with these findings, we observed no change in the accumulation of free $\alpha$-globin chain in red cells from bitopertin with DFP-treated $\beta$-thalassemic animals when compared with vehicle-treated animals (Supplemental Figure 6, D and E) or in total bilirubin, an indicator of chronic hemolysis (Supplemental Figure 6F).

These findings suggest that coadministration of DFP and bitopertin negates the beneficial effects of heme restriction induced by bitopertin alone. Bitopertin did not improve anemia in a pilot study in $\beta$-thalassemic patients, possibly because of the concomitant iron chelation therapy (31).

Bitopertin decreases the activity of the HRI with improvement of autophagy as a quality control process for $\mathrm{Hbb}^{\text {th } 3 /+}$ mouse erythroid cells. To understand the impact of bitopertin-induced heme restriction on heme-dependent regulatory pathways in erythropoiesis $(8,11)$, we evaluated the heme-dependent changes in the kinase activities of HRI. In the WT mice, treatment with bitopertin resulted in microcytic hypochromic anemia (Figure 1B and Supplemental Figure 1, A and B) characteristics of iron deficiency anemia, which requires 
HRI. In addition, HRI was activated with elevated protein expression in the spleen samples of WT mice treated with bitopertin (Supplemental Figure 7A). HRI protein expression was also increased and activated with hyperphosphorylation in bitopertin-treated mice as compared with control mice (Supplemental Figure 7A). These results agree with the decreased heme level in bitopertin-induced heme restriction, which activates HRI, leading to microcytic hypochromic anemia.

In $\beta$-thal mice, HRI is activated by accumulation of denatured $\alpha$-globin aggregates and oxidative stress from excessive heme (8). Because bitopertin markedly affects extramedullary erythropoiesis, erythroblasts from the spleen of $\mathrm{Hbb}^{\text {th } 3 /+}$ mice treated with bitopertin at the dosage of $10 \mathrm{mg} / \mathrm{kg} / \mathrm{d}$ were sorted for analysis of HRI activity. As shown in Figure 4A, bitopertin treatment reduced the hyperphosphorylation of highly activated HRI and the phosphorylation of eIF2 $\alpha$ in sorted splenic erythroblasts from $\beta$-thal mice. The reduced activity of HRI is consistent with the improvement of oxidative stress and the reduction in $\alpha$-globin aggregates in $\beta$-thal mice by bitopertin treatment (Figure 1C). Although HRI activity was reduced upon bitopertin treatment, it was still activated with significant eIF2 $\alpha$ phosphorylation (Figure $4 \mathrm{~A}$ ) to inhibit $\alpha$-globin protein synthesis and oxidative stress.

Recently, we reported a link between heme availability, HRI function, and autophagy during erythropoiesis (11). A previous study has shown that the autophagy gatekeeper mTOR is activated in $\beta$-thalassemic murine erythropoiesis, blocking autophagy with impairment of the quality control process involved in production of red cells (32). Given that efficient autophagy is required for erythroid maturation, we therefore evaluated the expression of some key proteins of autophagy in reticulocyte-enriched fractions from both mouse strains (33-35). We focused our analysis on Rab5, involved in maturation of autophagosome and recycling endosomes, and on specific markers of different trafficking compartments such as Lamp1, multivesicular bodies/late endosomes, or p62 as autophagy adapter controlling protein turnover. The latter has been previously shown to be an indirect marker of the efficiency of autophagic flux because p62 levels correlate with autophagy-related protein degradation $(33,35-40)$. In bitopertin-treated $\mathrm{Hbb}^{\text {th3/+}}$ mice, we found a reduction in mTOR complex 1 (mTORC1) activation (Ser2448) $(41,42)$ compared with vehicle-treated $H b b^{\text {th } 3 /+}$ mice (Figure 4B). This was associated with a reduction in accumulation of Rab5, Lamp1, and p62, indicating an improvement of autophagy in bitopertin-treated $\mathrm{Hbb}^{\text {th } 3 /+}$ mice (Figure 4B). Indeed, the improvement in the quality control process in the production of red cells was further supported by the reduction in (a) the amount of hemichromes bound to the red cell membrane (Figure 4C), (b) ROS levels (Figure 4D), (c) free $\alpha$ chains, and (d) the expression of key antioxidant systems such as Prx2 and its related enzyme, thioredoxin-reductase, where expression was increased in $\beta$-thalassemic red cells (Figure 4E, Supplemental Figure 7, A and B). This ends in the amelioration of red cell survival observed in bitopertin-treated $\beta$-thalassemic mice (Figure $4 \mathrm{~F}$ ).

Bitopertin affects in vitro human erythropoiesis. To explore the effect of bitopertin on human erythropoiesis, we tested bitopertin in vitro in $\mathrm{CD} 34^{+}$cells derived from peripheral blood of $\beta$-thalassemic intermedia patients and normal controls $(3,4,43)$. This culture system recapitulates the ineffective erythropoiesis observed in vivo in $\beta$-thal by exhibiting increased apoptosis and disturbed maturation $(3,4,43)$. Because bitopertin targets GlyT1, we evaluated the expression of this molecule in the early (7 days of culture) and in the late phase (14 days) of erythropoiesis. As shown in Figure 5A, the expression of GlyT1 was similar in both groups, and it was higher in the early phase of erythropoiesis.

We then carried out preliminary dose-response studies to identify the concentration of bitopertin to be tested in in vitro human $\beta$-thalassemic erythropoiesis. Because the amount of $\mathrm{CD} 34^{+}$cells derived from peripheral blood of patients with $\beta$-thalassemic intermedia is limited, the dose-response experiments were carried out only in cells from healthy volunteers. In preliminary experiments, various concentrations, timing, and frequencies of addition were tested for bitopertin, which resulted in the selection of a protocol using $10 \mathrm{nM}$ of bitopertin added at day 7 of the cell culture, a time at which GlyT1 reaches its peak (Supplemental Figure 8A). It is of note that an increase of annexin $\mathrm{V}^{+}$cells was observed at 14 days of cell culture only in the presence of $100 \mathrm{nM}$ bitopertin in normal erythroid precursors, suggesting a possible cell toxicity at this higher dose in this cell system (Supplemental Figure 8B).

The addition of $10 \mathrm{nM}$ bitopertin at day 7 on normal erythropoiesis showed a positive impact on cell viability at 14 days of cell culture (Supplemental Figure 8B). In normal erythropoiesis, bitopertin at $10 \mathrm{nM}$ induced a significant reduction in basophilic erythroblasts (Int-E) and an increase of orthochromatic erythroblasts (Late-E) (see Supplemental Figure 8C for gating strategy). Accordingly, an increase of apoptotic Int-E was noted in early erythropoiesis, while a significant reduction in apoptotic cells was observed in Late-E, overall indicating an acceleration of erythroid maturation of healthy cells (Supplemental Figure 9, A-D) $(4,44)$. 
A
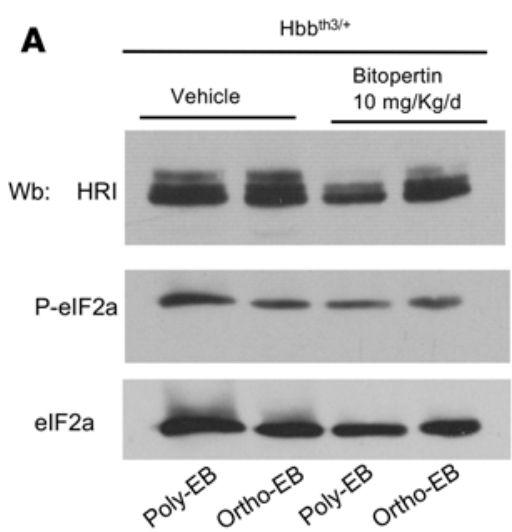
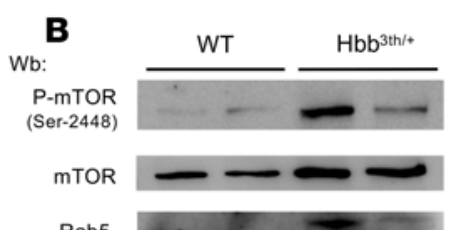

Rab5

Lamp1

p62

Catalase

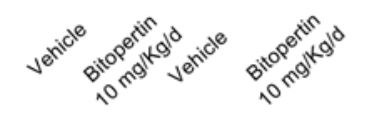

WT Vehicle

WT Bitopertin

$\square \mathrm{Hbb}^{3 \mathrm{H} /} \cdot$ Vehicle

口 $\mathrm{Hbb}^{3 \mathrm{H} / *}$ Bitopertin

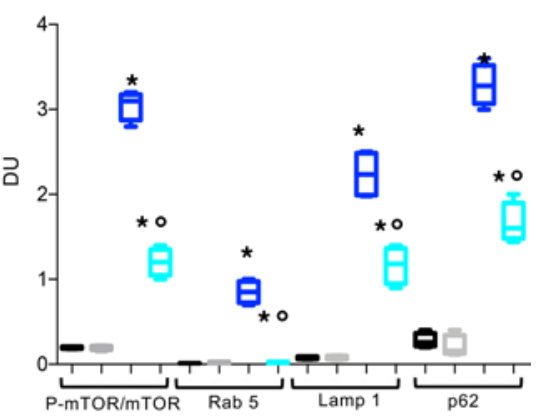

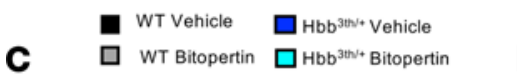

C WT Bitopertin $\square \mathrm{Hbb}^{3 \mathrm{HN}+}$ Bitopertin

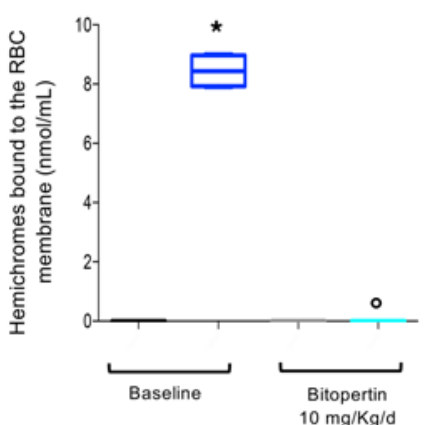

$\mathbf{F}$

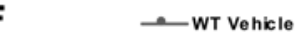

- Hbbth $3 /+$ Vehicle

- Hbbth $3 /+$ Bitopertin

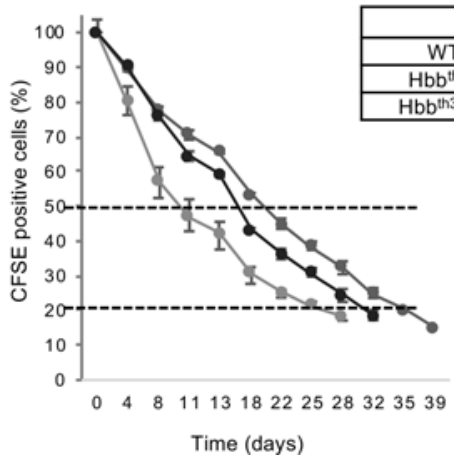

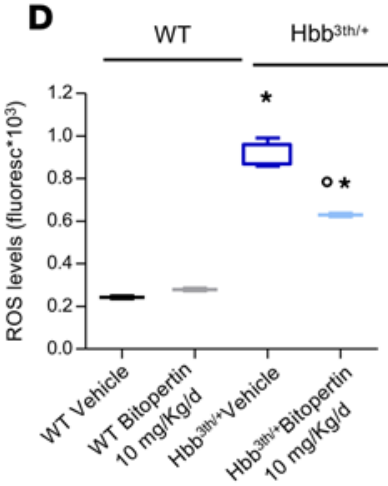

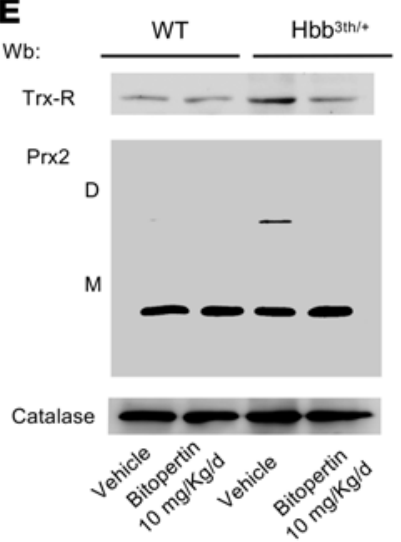

Figure 4. Bitopertin treatment modulates HRI activity and improves autophagy, resulting in optimization of the quality control process with amelio-

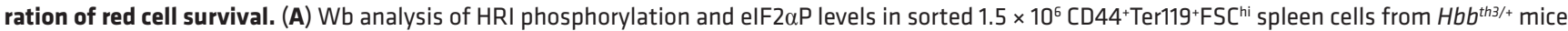
with and without bitopertin treatment (10 mg/kg/d, 28 days) $(n=3)$. (B) Left: Wb analysis of phospho-mTOR (p-mTOR), mTOR, Rab5, Lamp1, and p62 in reticulocyte-enriched fractions from WT and $\mathrm{Hbb}^{\text {thz/+ }}$ mice with and without bitopertin treatment $(n=3 ; 10 \mathrm{mg} / \mathrm{kg} / \mathrm{d}, 28$ days). Catalase was used as a protein loading control. Right: Densitometric analyses of the immunoblot bands similar to those shown are presented. Data are shown as median and minimum/maximum $(n=4)$; ${ }^{*} P<0.05$ compared with $W T ;{ }^{\circ} P<0.05$ compared with vehicle; 2 -way ANOVA with Holm-Šídák test for multiple comparisons. (C) Hemichromes bound to the membrane in WT and $\mathrm{Hbb}^{\text {th } 3 /+}$ mice with and without bitopertin treatment $(n=3 ; 10 \mathrm{mg} / \mathrm{kg} / \mathrm{d}, 28 \mathrm{days})$. Data are shown as median and minimum/maximum $(n=4) ; P<0.05$ compared with WT; ${ }^{\circ} P<0.05$ compared with vehicle; 2 -way ANOVA with Holm-Šídák test for multiple comparisons. (D) ROS levels in red cells of WT and $\mathrm{Hbb}^{\text {th } 3 /+}$ mice with and without bitopertin treatment (10 mg/kg/d, 28 days). Data are presented as median and minimum/maximum $(n=6) ;{ }^{*} P<0.05$ compared with vehicle-treated $H b b^{\text {th } 3 / 4}$ mice; ${ }^{\circ} \mathrm{P}<0.05$ compared with vehicle. 2 -way ANOVA with HolmŠídák test for multiple comparisons. (E) Wb analysis of thioredoxin-reductase (Trx-R) and peroxiredoxin-2 (Prx2) in erythrocytes from WT and $H b b^{\text {th } 3 /+}$ mice with and without bitopertin treatment ( $n=3 ; 10 \mathrm{mg} / \mathrm{kg} / \mathrm{d}, 28$ days). Catalase was used as a protein loading control (see Supplemental Figure 6, A and B, for densitometric analyses). (F) Red cell survival of CFSE-labeled red cells from WT and $\mathrm{Hbb}^{\text {th3/++}}$ mice with and without bitopertin treatment ( $n=3$; 10 $\mathrm{mg} / \mathrm{kg} / \mathrm{d}, 40$ days). Data are presented as mean $\pm \mathrm{SD}(n=3)$ from each group; ${ }^{*} P<0.05$ compared with WT; ${ }^{\circ} P<0.05$ compared with vehicle; Wilcoxon's signed-rank test for multiple comparisons. 
A

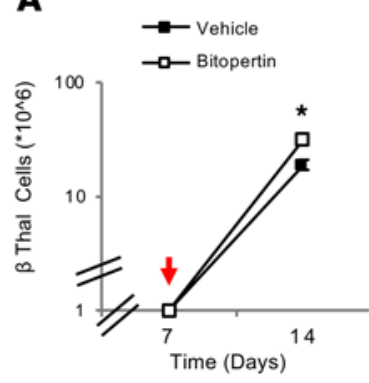

C
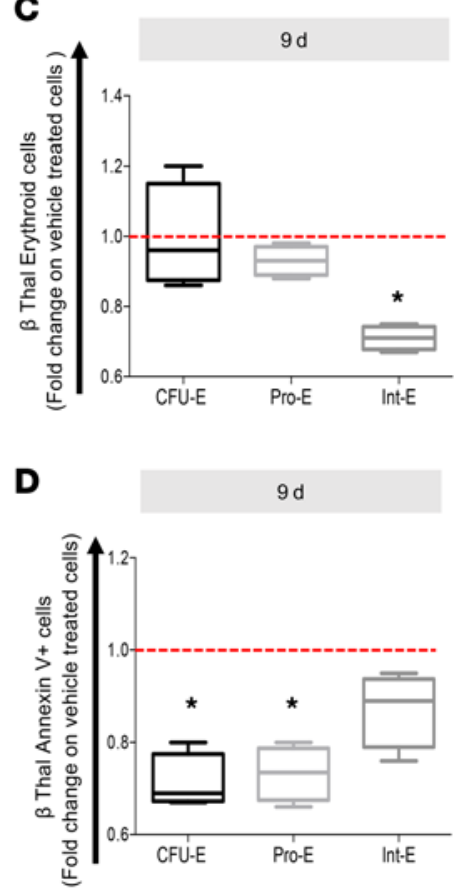

B

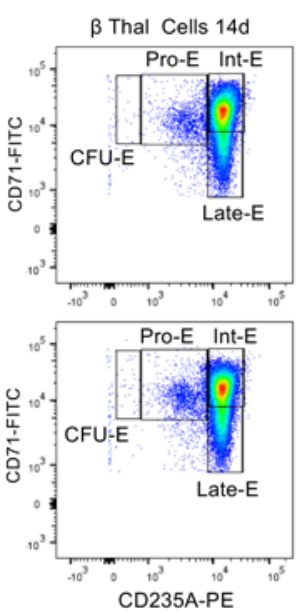

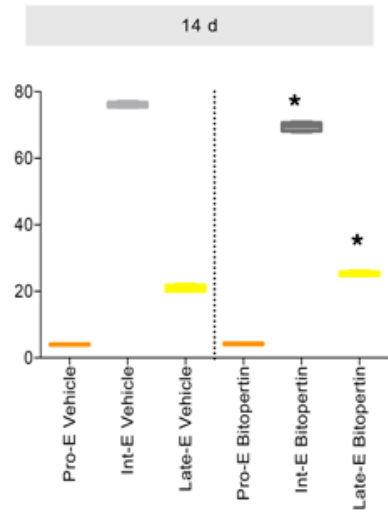

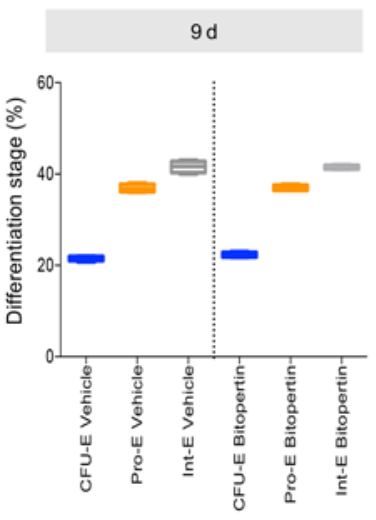

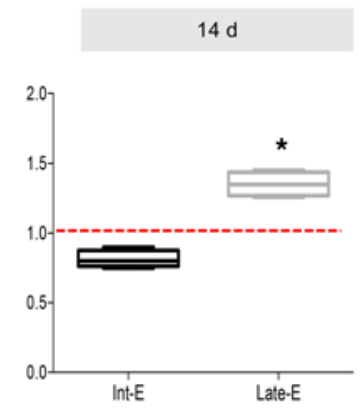
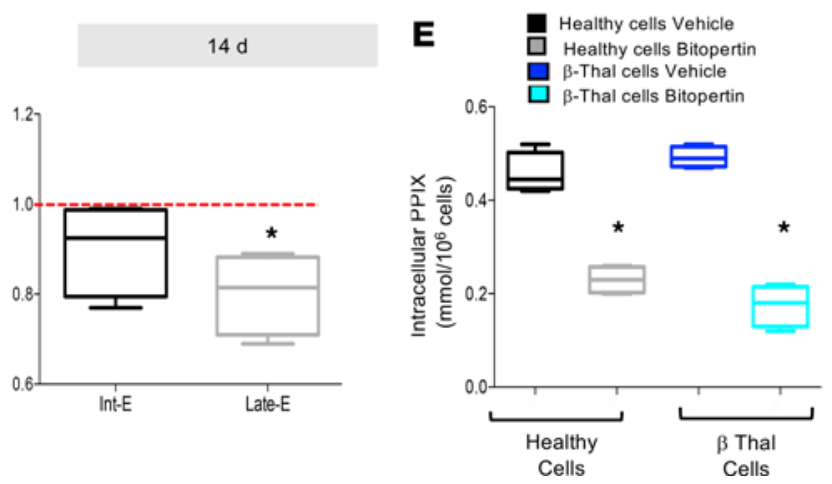

Figure 5. Bitopertin beneficially affects in vitro $\beta$-thalassemic erythropoiesis, with reduction of protoporphyrin IX, as a marker of heme restriction. (A) Cell proliferation of $\beta$-thalassemic erythroid precursors derived by in vitro liquid culture of $C D 34^{+}$cells isolated from peripheral blood of $\beta$-thal subjects with or without bitopertin $10 \mathrm{nM}(n=3)$. Arrows indicate when bitopertin $10 \mathrm{nM}$ was added to the culture medium (see Supplemental Figure 6B for dose-response data on cell viability in normal erythropoiesis). Data are presented as mean $\pm \mathrm{SD}$; ${ }^{*} P<0.05$ compared with vehicle-treated $\beta$-thal cells; Wilcoxon's signed-rank test for multiple comparisons. (B) Effect of bitopertin (10 $\mathrm{nM}$ ) on maturation pattern of $\beta$-thalassemic erythroid precursors from a single patient in triplicate. Representative flow cytometric plot and box plots of the differentiation pattern of $\beta$-thalassemic erythroid precursors at 14 days of culture with or without bitopertin (10 nM). The following surface markers were used: CD36, glycophorin-A, and CD71. This allows for the identification of the following homogenous cell populations: pro-erythroblasts (Pro-E), basophilic erythroblasts corresponding to intermediate erythroblasts (Int-E), and polychromatic and orthochromatic erythroblasts as late erythroblasts (Late-E) (see also refs. 4, 44). Data are shown as median and minimum/maximum $(n=4) ;{ }^{*} P<0.05$ compared with vehicle-treated $\beta$-thalassemic cells; Wilcoxon's signed-rank test for multiple comparisons. (C) Effect of bitopertin on the index of erythroid maturation of $\beta$-thalassemic cells from 4 separate individuals at 9 and 14 days of cell culture. ${ }^{*} P<0.05$ compared with vehicle-treated $\beta$-thalassemic cells; Mann-Whitney $U$ test with multiple-comparisons correction. (D) Effect of bitopertin (10 nM) on the amount of annexin $\mathrm{V}^{+}$cells during $\beta$-thalassemic erythroid maturation from 4 separate individuals at 9 and 14 days of cell culture. ${ }^{*} P<0.05$ compared with vehicle-treated $\beta$-thalassemic cells, Mann-Whitney $U$ test with multiple-comparison correction. (E) Intracellular content of protoporphyrin IX in healthy and $\beta$-thalassemic cells $\left(4.5 \times 10^{6}\right.$ cells) after 14 days of cell cultures with and without bitopertin $(10 \mathrm{nM})$. Data are presented as median and minimum/maximum ( $n=4)$ from each group; ${ }^{*} P$ $<0.05$ compared with vehicle; Mann-Whitney $U$ test with multiple-comparisons correction. 
In $\beta$-thalassemic erythropoiesis, bitopertin at $10 \mathrm{nM}$ induced a significant increase in cell viability compared with vehicle-treated cells (Figure 5A). This was associated with a marked decrease of basophilic erythroblasts (Int-E) and an increase of orthochromatic erythroblasts (Late-E) compared with vehicle-treated $\beta$-thalassemic cells (Figure 5, B and C). The improvement of $\beta$-thalassemic erythropoiesis was also supported by the significant reduction in the amounts of annexin $\mathrm{V}^{+}$cells in both early (CFU-E and pro-erythroblasts at 9 days) and late (orthochromatic erythroblasts at 14 days) phases of erythropoiesis (Figure 5D).

These data indicate that bitopertin, by promoting heme restriction, ameliorates human $\beta$-thalassemic erythropoiesis, accelerates erythroid maturation, and reduces cell apoptosis. The presence of a heme restriction state is supported by a reduction in protoporphyrin IX, a heme precursor, in bitopertin $\beta$-thalassemic erythroid cells (Figure 5E).

\section{Discussion}

Ineffective erythropoiesis and reduced red cell survival are the main determinants of the anemia of $\beta$-thal. Oxidant damage due to aggregated free $\alpha$ chain deposition on the cell membrane and to free heme is the main driver of the pathophysiology of the disease. A variety of strategies aimed at reducing oxidant damage or improving ineffective erythropoiesis have been considered, and a novel in silico model has been developed to test these interventions (45). A possible therapeutic approach is based on restriction of iron availability to reduced heme and free iron-induced oxidant damage. We have explored in this work the role of restricting the cellular supply of glycine with the use of bitopertin, an inhibitor of the GlyT1 transporter, because glycine is a key element for both heme synthesis and globin protein production.

In a mouse model of $\beta$-thal intermedia, we report here that the GlyT1 inhibitor, bitopertin, substantially improves anemia with a marked reduction in splenic erythropoiesis. In mice, splenic erythroid activity is rapidly activated in response to stress or in pathologic erythropoiesis $(1,46)$. Thus, we were not surprised to observe the reduction of spleen erythropoiesis with the improvement of ineffective erythropoiesis. Similar data were also observed in the same mouse model for $\beta$-thal treated with activin ligand trap RAP-011 (24). As summarized in Figure 6, bitopertin treatment significantly reduces $\beta$-thalassemic ineffective erythropoiesis accompanied by a reduction in heme level, based on a reduction of the heme precursor protoporphyrin IX free $\alpha$-globin aggregates, and oxidative stress, as well as the improvement of autophagy-based quality control processes. The reduction of heme levels upon bitopertin treatment is corroborated with the activation of HRI and the development of microcytic, hypochromic anemia in WT mice. HRI is responsible for microcytic and hypochromic hallmarks of iron deficiency anemia in iron and heme deficiency $(10,47)$. The beneficial effect of heme restriction upon bitopertin treatment in $\beta$-thal is most likely mediated by HRI. HRI is necessary to reduce phenotypic severity of $\beta$-thal, including proteotoxicity of $\alpha$-globin inclusions, ineffective erythropoiesis, and iron overload (47). However, activation of HRI alone in heme sufficiency cannot mitigate entirely the symptoms of $\beta$-thal. Reduction of heme by bitopertin provides an additional means of reducing oxidative stress. In heme deficiency, HRI inhibits not only globin protein synthesis but also heme biosynthesis as demonstrated in ferrochelatase-deficient mice (47). Furthermore, HRI is activated and induces activating transcription factor 4 (ATF4) mRNA translation in mouse $\beta$-thalassemic erythroid precursors $(47,48)$. Activation of ATF4 by HRI is necessary to mitigate oxidative stress in iron deficiency, which leads to heme deficiency (11). Recent global assessment of gene expression in erythroblasts demonstrates that HRI and heme-mediated translation and subsequent transcriptome changes are most highly activated during iron/heme deficiency (49). The increased mTORC1 activity in $\beta$-thal mice is most likely due to an elevated EPO level in the presence of anemia. HRI is necessary to repress EPO/mTORC1 signaling as a feedback mechanism for EPO-stimulated erythropoiesis in iron deficiency to prevent ineffective erythropoiesis (11), which also occurs in $\beta$-thal. Repression of mTORC1 by HRI can also mitigate autophages.

Heme plays a key role in normal erythropoiesis and in the alterations observed in $\beta$-thal erythropoiesis $(3,5,17)$. We and others have shown biphasic behavior in heme biosynthesis, characterized by heme accumulation in the early phase of $\beta$-thal erythropoiesis, followed by a relative heme reduction in the late phase of $\beta$-thal erythropoiesis (3). Thus, the pool of "uncommitted heme" in erythroblasts during $\beta$-thal erythropoiesis is not fixed but varies dynamically according to the development state of erythroblasts $(3,5$, 17). In erythroblasts treated with bitopertin, the reduction of protoporphyrin IX associated with increased $\mathrm{Hb}$ supports the importance of heme restriction to optimize cell hemoglobinization (Figure 5E). 


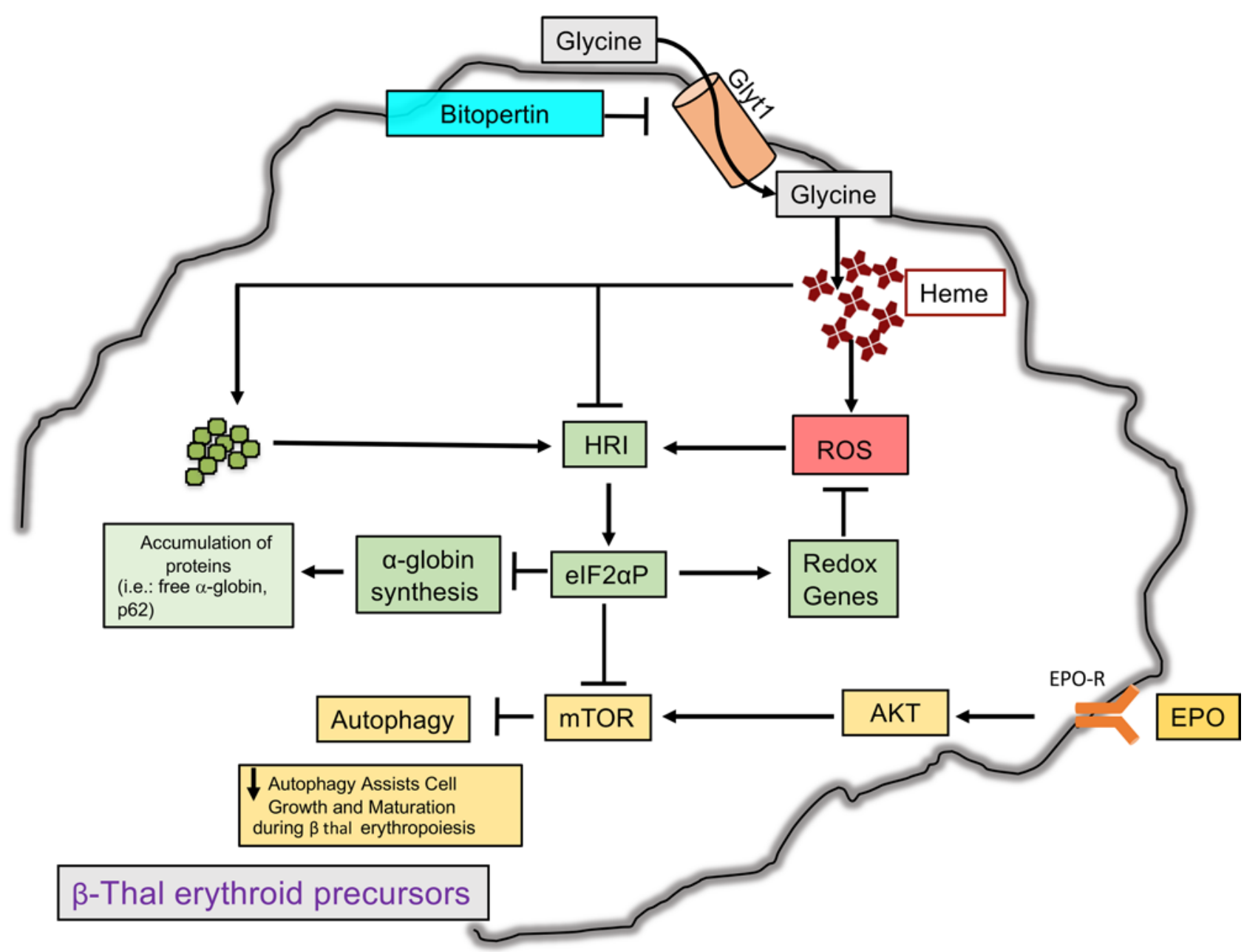

Figure 6. Schematic diagram of the downstream effects on $\beta$-thalassemic erythroblasts when glycine uptake is impaired by bitopertin, a specific inhibitor of the GlyT1 transporter. Bitopertin modulates heme biosynthesis, which affects the amount of free heme (in the in vitro model of human erythropoiesis, we observed a reduction of protoporphyrin IX). This results in reduction of oxidative stress with associated decrease in activation of HRI, which orchestrates elF $2 \alpha$ and senses free heme concentration. The optimization between heme and $\alpha$-globin chain synthesis further contributes to the reduction in generation of ROS, resulting in the blockage of mTOR and activation of autophagy, which contributes to the clearance of damaged proteins, assisting $\beta$-thalassemic cell survival during growth and maturation.

The data in $\beta$-thal mouse treated with low $(3-10 \mathrm{mg} / \mathrm{kg} / \mathrm{d})$ and high $(30 \mathrm{mg} / \mathrm{kg} / \mathrm{d})$ dosages of bitopertin provide some insights into how higher dosages affect the iron regulatory system and possibly help in preventing excessive iron restriction.

A general concern regarding the use of iron restriction as a therapeutic strategy for $\beta$-thal is the establishment of safe dose ranges and conditions, to prevent an excessive restriction of iron, which will negate the original therapeutic benefits. Similar experiences have been reported in $\beta$-thalassemic mice treated with either mini-hepcidin or apo-transferrin or with molecules interfering with Tmprss6 function $(6,28,50-53)$. A "sweet spot" seems to exist between iron restriction and iron deficiency induced by one of the abovementioned treatments, and a deviation from this optimal point may negate the amelioration of $\beta$-thalassemic erythropathology by superimposing a clinically significant iron-restricted/iron-deficient erythropoiesis. Our in vitro data with $\mathrm{CD} 34^{+}$erythroid precursors from patients with $\beta$-thal do not allow us to establish dose ranges for the human studies, with cellular toxicity being observed with $100 \mathrm{nM}$ bitopertin (Supplemental Figure 7B), but show similar reduction in ineffective erythropoiesis markers to the one observed in the $\mathrm{Hbb}^{\text {th } 3 /+}$ mouse model with a dose of $10 \mathrm{nM}$, suggesting that the experimental data of the mouse model can be extrapolated to humans.

Because iron chelation is currently indicated for all $\beta$-thal syndromes requiring therapeutic intervention, we examined in the $H b b^{t h 3 /+}$ mice the effects of bitopertin treatment in conjunction with the iron chelator DFP. Prior work by Casu et al. showed no deleterious effect of DFP administration to $H b b^{\text {th }} /+$ 
mice (28). Our data indicate that concomitant iron chelation with DFP in the $H b b^{t h 3 /+}$ mice severely blunts or negates the therapeutic benefits of bitopertin (Figure 3). Worsening anemia and microcytosis in $\mathrm{Hbb}^{\text {th } 3 /+}$ mice treated with both bitopertin and DFP may indicate an unacceptably high level of iron restriction, negatively affecting $\beta$-thal erythropoiesis.

Preliminary findings of a phase II trial of bitopertin in patients with transfusion-dependent $\beta$-thal have been recently reported in an abstract (31). With the dosages used in this study, a reduction in $\mathrm{Hb}$ and $\mathrm{MCH}$ were noted with no improvement in hemolysis. The study tested a dose escalation protocol (30 to 60 to 90 $\mathrm{mg} / \mathrm{d}$ ) for a treatment duration of 16 weeks, and it may be postulated that the concomitant iron chelation therapy could have affected the hematologic response, as we have shown for a $\beta$-thalassemic mouse model.

In conclusion, we show that bitopertin-induced inhibition of the GlyT1 transporter beneficially affects $\beta$-thal ineffective erythropoiesis and improves anemia, hemolysis, and red cell survival in $H b b^{\text {th } 3 /+}$ mice. Multiple markers in different tissues indicated reduced oxidant damage, reduced liver and spleen iron overload, and improved autophagy assisting erythroid maturation. The ineffective erythropoiesis observed with in vitro culture of human $\beta$-thal precursor shows similar improvement with bitopertin exposure, indicating the translatability of effects on erythropoiesis between a mouse model and humans.

\section{Methods}

Mouse strains and design of the study. Female 2-month-old WT (C57BL/6) and Hbb th3/+ mice (from established mouse colony in CIRSAL, University of Verona) were studied. In mouse strains, hematologic parameters, red cell indices, and reticulocyte count were evaluated at baseline and at different time points as previously reported $(15,54)$. Blood was collected with retroorbital venipuncture in anesthetized mice using heparinized microcapillary tubes. Hematologic parameters were evaluated on a Siemens ADVIA 2120 hematology analyzer. Hematocrit and $\mathrm{Hb}$ were manually determined $(4,55)$. We determined $\alpha$-globin membrane aggregates and soluble $\mathrm{Hb}$ as described by Dussit et al. (24). Determining the amount of hemichromes bound to the red cell membrane was carried out as previously reported $(15,56)$

Flow cytometric analysis of mouse erythroid precursors and molecular analysis of sorted erythroid cells. Flow cytometric analysis of erythroid precursors from bone marrow and spleen from WT and $\mathrm{Hbb}^{\text {th } 3 /+}$ mice was carried out as previously described using the CD44-Ter119 or CD71-Ter119 strategy $(1,12,57)$. Analysis of apoptotic basophilic, polychromatic, and orthochromatic erythroblasts was carried out on the CD44-Ter119 gated cells using the Annexin V PE Apoptosis Detection Kit (eBioscience), following the manufacturer's instructions. Erythroblasts' ROS values were measured as previously reported by Matte et al. (12). Sorted cells were used for (a) morphologic analysis of erythroid precursors on cytospin preparations stained with May-Grünwald-Giemsa, (b) qRT-PCR analysis, and (c) immunoblot analysis. Details of qRT-PCR protocol and immunoblot used for the analysis of sorted erythroblasts and red cells are described in Supplemental Methods.

HRI assay. HRI assay was carried out as previously reported on sorted erythroblasts from the spleen of $H b b^{t h 3 /+}$ mice treated with either vehicle or bitopertin (11).

Red cell survival. Red cells from WT and $\beta$-thal mice treated with or without bitopertin were labeled with CFSE (10 $\mu \mathrm{M}$; Molecular Probes, Invitrogen, Thermo Fisher Scientific) in PBS, with 0.5\% BSA, for 20 minutes at $37^{\circ} \mathrm{C}$ as previously reported (13).

Pearl's analysis of liver and spleen. Immediately following dissection, the spleen and liver were formalin-fixed and paraffin-embedded for Pearl's staining (12).

Molecular analysis of liver. Protocols used for RNA isolation, cDNA preparation, and qRT-PCR have been previously described (58). Detailed primer sequences are available on request and shown in Supplemental Table 1. Liver immunoblot analysis was performed as previously described $(12,59)$. Oxidized proteins were monitored using OxyBlot Protein Oxidation Detection Kit (MilliporeSigma) as previously reported (12).

Studies on in vitro human erythropoiesis. We analyzed 10 erythroid cultures obtained from the peripheral blood of different normal subjects and 10 erythroid cultures obtained from 4 patients with homozygous $\beta$-thalassemic intermedia $\left(\beta^{0 \operatorname{cod} 39}\right)(4)$. The erythroid cell antigen profiling and the sorting of erythroid precursors were carried out as previously described (4). Immunoblot analysis of human erythroid precursors was carried out using $1 \times 10^{6}$ cells at 7 and 14 days of culture from both normal and $\beta$-thalassemic subjects, which were solubilized as previously described (4). Determination of protophorphyrin IX was carried out on cells from healthy donors in the presence and the absence of bitopertin. Details are reported in Supplemental Methods (60-64). 
Statistics. Data were analyzed using 2-way ANOVA for repeated measures between the mice of various genotypes as well as between healthy and thalassemic cells. A difference with $P<0.05$ was considered significant.

Study approval. The Institutional Animal Experimental Committee of University of Verona and the Italian Ministry of Health (Rome, Italy) approved the experimental protocols. Informed consent for the use of their blood samples was obtained from healthy subjects and patients.

\section{Author contributions}

AM designed and carried out the experiments and analyzed data; LDF and CB designed the experiments, analyzed the data, and wrote the manuscript; EF carried out immunoblot experiments and analyzed the data; EB carried out CD34-derived liquid cultures and analyzed data; MLDP carried out the protophorphyrin IX experiments and analyzed data; LD and IA carried out qRT-PCR analyses; AI contributed to analyzing data and writing the manuscript; AMG and JJC carried out HRI experiments, discussed data, and edited the manuscript; AJ and CL carried out blinded pathologic analyses and contributed to writing the manuscript; FT carried out the analyses of hemichromes and analyzed data; and MW, AK, AH, NM, and TT contributed to experimental design, data analysis, and manuscript writing.

\section{Acknowledgments}

This paper was supported by a Roche research collaborative grant (EPBA1834150) to LDF.

Address correspondence to: Carlo Brugnara, Boston Children's Hospital, Department of Laboratory Medicine, 300 Longwood Avenue, BA 760; Boston, Massachusetts 02115, USA. Phone: 617.355.6610; Email: carlo.brugnara@childrens.harvard.edu.

1. Liu J, et al. Quantitative analysis of murine terminal erythroid differentiation in vivo: novel method to study normal and disordered erythropoiesis. Blood. 2013;121(8):e43-e49.

2. An X, et al. Global transcriptome analyses of human and murine terminal erythroid differentiation. Blood. 2014;123(22):34663477 .

3. De Franceschi L, et al. Oxidative stress modulates heme synthesis and induces peroxiredoxin- 2 as a novel cytoprotective response in $\beta$-thalassemic erythropoiesis. Haematologica. 2011;96(11):1595-1604.

4. Franco SS, et al. Resveratrol accelerates erythroid maturation by activation of $\mathrm{FoxO} 3$ and ameliorates anemia in beta-thalassemic mice. Haematologica. 2014;99(2):267-275.

5. De Franceschi L, et al. Oxidative stress and $\beta$-thalassemic erythroid cells behind the molecular defect. Oxid Med Cell Longev. 2013;2013:985210.

6. Rivella S. $\beta$-thalassemias: paradigmatic diseases for scientific discoveries and development of innovative therapies. Haematologica. 2015;100(4):418-430.

7. Rund D, Rachmilewitz E. Beta-thalassemia. N Engl J Med. 2005;353(11):1135-1146.

8. Chen JJ. Regulation of protein synthesis by the hemeregulated eIF2alpha kinase: relevance to anemias. Blood. 2007;109(7):2693-2699.

9. Lu L, Han AP, Chen JJ. Translation initiation control by hemeregulated eukaryotic initiation factor 2alpha kinase in erythroid cells under cytoplasmic stresses. Mol Cell Biol. 2001;21(23):7971-7980.

10. Han AP, et al. Hemeregulated eIF2alpha kinase (HRI) is required for translational regulation and survival of erythroid precursors in iron deficiency. EMBO J. 2001;20(23):6909-6918.

11. Zhang S, Macias-Garcia A, Velazquez J, Paltrinieri E, Kaufman RJ, Chen JJ. HRI coordinates translation by eIF2 $\alpha$ P and mTORC1 to mitigate ineffective erythropoiesis in mice during iron deficiency. Blood. 2018;131(4):450-461.

12. Matte A, et al. Peroxiredoxin-2: a novel regulator of iron homeostasis in ineffective erythropoiesis. Antioxid Redox Signal. 2018;28(1):1-14.

13. Matte A, et al. The interplay between peroxiredoxin- 2 and nuclear factor-erythroid 2 is important in limiting oxidative mediated dysfunction in $\beta$-thalassemic erythropoiesis. Antioxid Redox Signal. 2015;23(16):1284-1297.

14. Matte A, et al. Membrane association of peroxiredoxin-2 in red cells is mediated by the $\mathrm{N}$-terminal cytoplasmic domain of band 3. Free Radic Biol Med. 2013;55:27-35.

15. Matte A, et al. Peroxiredoxin-2 expression is increased in beta-thalassemic mouse red cells but is displaced from the membrane as a marker of oxidative stress. Free Radic Biol Med. 2010;49(3):457-466.

16. Garcia-Santos D, Schranzhofer M, Bergeron R, Sheftel AD, Ponka P. Extracellular glycine is necessary for optimal hemoglobinization of erythroid cells. Haematologica. 2017;102(8):1314-1323.

17. Garcia-Santos D, et al. Inhibition of heme oxygenase ameliorates anemia and reduces iron overload in a $\beta$-thalassemia mouse model. Blood. 2018;131(2):236-246.

18. Wolosker H. NMDA receptor regulation by D-serine: new findings and perspectives. Mol Neurobiol. 2007;36(2):152-164.

19. Eulenburg V, Armsen W, Betz H, Gomeza J. Glycine transporters: essential regulators of neurotransmission. Trends Biochem Sci. 2005;30(6):325-333.

20. Winter M, et al. Effects of GlyT1 inhibition on erythropoiesis and iron homeostasis in rats. Exp Hematol. 2016;44(10):964-974.e4

21. Umbricht $\mathrm{D}$, et al. Effect of bitopertin, a glycine reuptake inhibitor, on negative symptoms of schizophrenia: a randomized, 
double-blind, proof-of-concept study. JAMA Psychiatry. 2014;71(6):637-646.

22. Black MD, et al. Procognitive and antipsychotic efficacy of glycine transport 1 inhibitors (GlyT1) in acute and neurodevelopmental models of schizophrenia: latent inhibition studies in the rat. Psychopharmacology (Berl). 2009;202(1-3):385-396.

23. Roberts BM, Shaffer CL, Seymour PA, Schmidt CJ, Williams GV, Castner SA. Glycine transporter inhibition reverses ketamine-induced working memory deficits. Neuroreport. 2010;21(5):390-394.

24. Dussiot M, et al. An activin receptor IIA ligand trap corrects ineffective erythropoiesis in $\beta$-thalassemia. Nat Med. 2014;20(4):398-407.

25. Kautz L, Jung G, Valore EV, Rivella S, Nemeth E, Ganz T. Identification of erythroferrone as an erythroid regulator of iron metabolism. Nat Genet. 2014;46(7):678-684.

26. Kautz L, Jung G, Nemeth E, Ganz T. Erythroferrone contributes to recovery from anemia of inflammation. Blood. 2014;124(16):2569-2574.

27. Kautz L, Nemeth E. Molecular liaisons between erythropoiesis and iron metabolism. Blood. 2014;124(4):479-482.

28. Casu C, et al. Minihepcidin peptides as disease modifiers in mice affected by $\beta$-thalassemia and polycythemia vera. Blood. 2016;128(2):265-276.

29. Nai A, et al. Limiting hepatic Bmp-Smad signaling by matriptase-2 is required for erythropoietin-mediated hepcidin suppression in mice. Blood. 2016;127(19):2327-2336.

30. Aschemeyer S, Gabayan V, Ganz T, Nemeth E, Kautz L. Erythroferrone and matriptase-2 independently regulate hepcidin expression. Am J Hematol. 2017;92(5):E61-E63.

31. Taher A, et al. Phase 2 clinical trial results for bitopertin, an oral glycine transporter 1 inhibitor, in patients with non-transfusion dependent beta-thalassemia. Blood. 2018;132(Supp1_1):3635.

32. Zhang X, et al. FOXO3-mTOR metabolic cooperation in the regulation of erythroid cell maturation and homeostasis. Am J Hematol. 2014;89(10):954-963.

33. Lupo F, et al. A new molecular link between defective autophagy and erythroid abnormalities in chorea-acanthocytosis. Blood 2016;128(25):2976-2987.

34. Zhang J, et al. Mitochondrial clearance is regulated by Atg7-dependent and -independent mechanisms during reticulocyte maturation. Blood. 2009;114(1):157-164.

35. Beneduce E, et al. Fyn kinase is a novel modulator of erythropoietin signaling and stress erythropoiesis. Am J Hematol. 2019;94(1):10-20.

36. Komatsu M, et al. The selective autophagy substrate p62 activates the stress responsive transcription factor Nrf2 through inactivation of Keap1. Nat Cell Biol. 2010;12(3):213-223.

37. Jiang T, Harder B, Rojo de la Vega M, Wong PK, Chapman E, Zhang DD. p62 links autophagy and Nrf2 signaling. Free Radic Biol Med. 2015;88(Pt B):199-204

38. Bechtel W, et al. Vps34 deficiency reveals the importance of endocytosis for podocyte homeostasis. J Am Soc Nephrol. 2013;24(5):727-743

39. Klionsky DJ, et al. Guidelines for the use and interpretation of assays for monitoring autophagy. Autophagy. 2012;8(4):445-544.

40. Bartlett BJ, et al. p62, Ref(2)P and ubiquitinated proteins are conserved markers of neuronal aging, aggregate formation and progressive autophagic defects. Autophagy. 2011;7(6):572-583.

41. Tan P, et al. The PI3K/Akt/mTOR pathway regulates the replicative senescence of human VSMCs. Mol Cell Biochem. 2016;422(1-2):1-10.

42. Chiang GG, Abraham RT. Phosphorylation of mammalian target of rapamycin (mTOR) at Ser-2448 is mediated by p70S6 kinase. J Biol Chem. 2005;280(27):25485-25490.

43. De Franceschi L, et al. K-CL co-transport plays an important role in normal and beta thalassemic erythropoiesis. Haematologica. 2007;92(10):1319-1326.

44. Merryweather-Clarke AT, et al. Global gene expression analysis of human erythroid progenitors. Blood. 2011;117(13):e96-108

45. Mazer NA, Hermosilla R, Koerner A. Quantifying the pathophysiology of beta-thalassemia using a novel in-silico model of red blood cell production and lifespan. Blood. 2017;130(Suppl_1):2227.

46. Guihard S, et al. The MAPK ERK1 is a negative regulator of the adult steady-state splenic erythropoiesis. Blood. 2010;115(18):3686-3694.

47. Han AP, Fleming MD, Chen JJ. Hemeregulated eIF2alpha kinase modifies the phenotypic severity of murine models of erythropoietic protoporphyria and beta-thalassemia. J Clin Invest. 2005;115(6):1562-1570.

48. Suragani RN, et al. Hemeregulated eIF2 $\alpha$ kinase activated Atf4 signaling pathway in oxidative stress and erythropoiesis. Blood. 2012;119(22):5276-5284.

49. Zhang S, et al. HRI coordinates translation necessary for protein homeostasis and mitochondrial function in erythropoiesis. Elife. 2019;8:e46976

50. Li H, et al. Transferrin therapy ameliorates disease in beta-thalassemic mice. Nat Med. 2010;16(2):177-182.

51. Nai A, et al. Deletion of TMPRSS6 attenuates the phenotype in a mouse model of $\beta$-thalassemia. Blood. 2012;119(21):5021-5029.

52. Guo S, et al. Inactivation of specific $\beta$ cell transcription factors in type 2 diabetes. J Clin Invest. 2013;123(8):3305-3316.

53. Schmidt PJ, et al. An RNAi therapeutic targeting Tmprss6 decreases iron overload in Hfe(-/-) mice and ameliorates anemia and iron overload in murine $\beta$-thalassemia intermedia. Blood. 2013;121(7):1200-1208.

54. de Franceschi L, et al. In vivo reduction of erythrocyte oxidant stress in a murine model of beta-thalassemia. Haematologica. 2004;89(11):1287-1298.

55. Dalle Carbonare L, et al. Hypoxia-reperfusion affects osteogenic lineage and promotes sickle cell bone disease. Blood. 2015;126(20):2320-2328.

56. Matté A, et al. The novel role of peroxiredoxin-2 in red cell membrane protein homeostasis and senescence. Free Radic Biol Med. 2014; $76: 80-88$

57. Konstantinidis DG, et al. Cytokinesis failure in RhoA-deficient mouse erythroblasts involves actomyosin and midbody dysregulation and triggers p53 activation. Blood. 2015;126(12):1473-1482.

58. Petrillo S, et al. Heme accumulation in endothelial cells impairs angiogenesis by triggering paraptosis. Cell Death Differ. 
2018;25(3):573-588.

59. Kalish BT, et al. Dietary $\omega-3$ fatty acids protect against vasculopathy in a transgenic mouse model of sickle cell disease. Haematologica. 2015;100(7):870-880.

60. Piomelli S. Free erythrocyte porphyrins in the detection of undue absorption of $\mathrm{Pb}$ and of Fe deficiency. Clin Chem. 1977;23(2):264-269.

61. Chisolm J, Brown DH. Micro-scale photofluorometric determination of "free erythrocyte pophyrin" (protoporphyrin IX). Clin Chem. 1975;21(11):1669-1682.

62. Bradford MM. A rapid and sensitive method for the quantitation of microgram quantities of protein utilizing the principle of protein-dye binding. Anal Biochem. 1976;72:248-254.

63. Sorianello EM, Mazzetti MB. Simple and rapid method for the determination of coproporphyrinogen oxidase activity. $J$ Biochem Biophys Methods. 2000;45(1):75-86.

64. Macours P, Cotton F. Improvement in HPLC separation of porphyrin isomers and application to biochemical diagnosis of porphyrias. Clin Chem Lab Med. 2006;44(12):1433-1440. 